\title{
Coherent scattering function in the reptation model: Analysis beyond asymptotic limits
}

\author{
Lothar Schäfer, ${ }^{1}$ Ute Ebert, ${ }^{2}$ and Artur Baumgärtner ${ }^{3}$ \\ ${ }^{1}$ Universität GH Essen, Universitätsstrasse 5, 45117 Essen, Germany \\ ${ }^{2}$ Centrum voor Wiskunde en Informatica, P.O. Box 94079, 1090 GB Amsterdam, The Netherlands \\ ${ }^{3}$ Institut für Festkörperforschung, Forschungszentrum Jülich, 52425 Jülich, Germany
}

(Received 5 January 2002; published 21 June 2002)

\begin{abstract}
We calculate the coherent dynamical scattering function $S_{c}(q, t ; N)$ of a flexible chain of length $N$, diffusing through an ordered background of topological obstacles. As an instructive generalization, we also calculate the scattering function $S_{c}(q, t ; M, N)$ for the central piece of length $M \leqslant N$ of the chain. Using the full reptation model, we treat global creep, tube length fluctuations, and internal relaxation within a consistent and unified approach. Our theory concentrates on the universal aspects of reptational motion, and our results in all details show excellent agreement with our simulations of the Evans-Edwards model, provided we allow for a phenomenological prefactor which accounts for nonuniversal effects of the microstructure of the Monte Carlo chain, present for short times. Previous approaches to the coherent structure function can be analyzed as special limits of our theory. First, the effects of internal relaxation can be isolated by studying the limit $N \rightarrow \infty, M$ fixed. The results do not support the model of a "Rouse chain in a tube." We trace this back to the nonequilibrium initial conditions of the latter model. Second, in the limit of long chains $(M=N \rightarrow \infty)$ and times large compared to the internal relaxation time $\left(t / N^{2} \rightarrow \infty\right)$, our theory reproduces the results of the primitive chain model. This limiting form applies only to extremely long chains, and for chain lengths accessible in practice, effects of, e.g., tube length fluctuations are not negligible.
\end{abstract}

DOI: 10.1103/PhysRevE.65.061505

PACS number(s): 82.35.Lr, 05.40.-a

\section{INTRODUCTION}

The equilibrium dynamics of a dense polymer system, i.e., a melt, a solution of high concentration, or a free chain moving through a gel, is an important topic of polymer physics. It has been investigated for many years, but is still not fully understood. The problem is quite complex, even if we concentrate on the motion of a single chain. Clearly, its motion is strongly hindered by the surrounding chains, which the chain considered cannot cross. This has led to the idea [1] that the motion of the chain is confined to a tube roughly defined by its instantaneous configuration. Thus the tube is assumed to have a random walk configuration, which changes only by the motion of the chain ends. The ends can retract into the tube which thus is effectively shortened, and they can creep out of the original tube, thus creating a new tube segment in some random direction. The interior parts of the tube are assumed to be fixed in space until they are reached by the diffusive motion of the chain ends. This concept of a tube is one basic ingredient of the "reptation" [1,2] scenario, which certainly is valid provided the obstacles confining the chain motion form a rigid, time independent network. In a realistic system the surrounding chains are mobile, which sheds some doubt on the postulated existence of a well defined tube. Indeed, there exist other approaches $[3,4]$, more in line with standard many body theory, which are not based on the tube concept.

Most work on the reptation model concentrates on asymptotic results expected to hold for long chains in special time regions (see Sec. II A). In comparison to experiments or simulations, these results often fail at a quantitative level [5], and partly other theories seem to be more satisfactory $[3,4]$. Thus some work [6] has been invested to incorporate additional physical effects such as relaxation of the surrounding or specific interaction effects into asymptotic reptation theory. However, the evaluation of the pure reptation model outside asymptotic limits has found only little attention. In recent work $[7,8]$, we presented such a calculation for the motion of individual segments of the chain. We found that asymptotic results, which for the quantities considered take the form of specific power laws, apply only to surprisingly long chains. Large time intervals are covered by crossover regions. Our crossover functions compare very well to simulations [9] of the pure reptation model, i.e., to the motion of a flexible chain through a fixed regular lattice of impenetrable obstacles. Furthermore, also results of simulations of melts look quite similar to our analytical results for short chains. This is consistent with the observation that the tube diameter, i.e., the average distance among effective obstacles extracted from the simulations, is fairly large. Since in the pure reptation model, the tube width is of the order of the effective segment size of the reptating chain, this implies that to map melt dynamics on the reptation model, we have to consider a coarse grained chain of effective segment number $N / N_{e}$. Here $N$ is the chain length (polymerization index) of the physical macromolecule, whereas the "entanglement length" $N_{e}$ is the length of a subchain that shows a coil radius of the order of the obstacle spacing. In recent simulations [10] of melts, a value $N / N_{e} \approx 14$ was reached, far below the value $N / N_{e} \gtrsim 50$ needed according to our theory to clearly identify asymptotic power laws. Other recent simulations [11] reach a value $N / N_{e} \approx 300$, but for this chain length they cover only times short compared to the characteristic time scales of the reptation model. Still, the onset of a first power law regime is seen, again consistent with our theory. Thus, concerning the motion of individual chain segments within a melt, there at present seems to be no need to invoke other mechanisms than pure reptation. 
The motion of specific segments is easily accessible only to computer experiments. Physical experiments often measure dynamic scattering functions. Asymptotic results of the reptation model for the coherent scattering function have been worked out previously $[12,13,2]$, but our analysis of segment motion suggests that an evaluation of the scattering function outside asymptotic limits is needed. This is the topic of the present work. We use the same analytical reptation model as in our previous analysis [8]. We also measured the coherent structure function in Monte Carlo simulations, again using the same implementation of the model as previously [9]. This allows for a comparison among theory and data, where all parameters are fixed by our previous work. Some results of the simulations will be presented here, but a detailed comparison of our simulation results to the present and previous theories will be presented in a separate, less technical paper [14].

In the following section, we briefly review the basic features of the reptation model and recall previous results for the coherent structure function. In Sec. III, we introduce our analytical model and outline the structure of our approach. In Sec. IV, we consider those contributions to the coherent structure function in which the initial tube is not yet completely destroyed by the stochastic motion. A rigorous analysis is possible as long as end effects can be neglected. These end effects, known as "tube renewal" and "tube length fluctuations," can be treated only in some approximation. We here generalize an approach that in our previous work gave good results for the segment motion. In Sec. V, we compare our rigorous results for the motion within the initial tube to those of the model of a "Rouse chain in a coiled tube" [12]. Pronounced differences are found and their origin is clarified. In Sec. VI, we derive an integral equation which takes complete tube destruction into account. For long chains and times large compared to the internal relaxation time of the chain, we recover the results of the "primitive chain" model $[13,2]$, as shown in Sec. VII. Typical numerical results of our theory are discussed in Sec. VIII. It is found that tube length fluctuations, which have been neglected in previous calculations of the coherent scattering function, in fact determine the scattering up to times larger than the Rouse time. In Sec. VIII, we also present some results of our simulations, which compare favorably with our theory. Finally, Sec. IX contains a summary and conclusions. The full evaluation of the reptation model leads to quite involved expressions, and some part of the analysis is summarized in Appendixes.

\section{REVIEW OF THE REPTATION SCENARIO AND OF PREVIOUS RESULTS FOR THE COHERENT STRUCTURE FUNCTION}

\section{A. Basic dynamics and time scales}

As mentioned in the Introduction, the reptation model assumes the existence of a tube defined by the instantaneous configuration of the chain together with the surrounding obstacles. The chain cannot leave the tube sideways since it would have to fold into a double-stranded conformation, which costs too much entropy. Those parts of the chain, which lie stretched in the tube, essentially cannot move. In

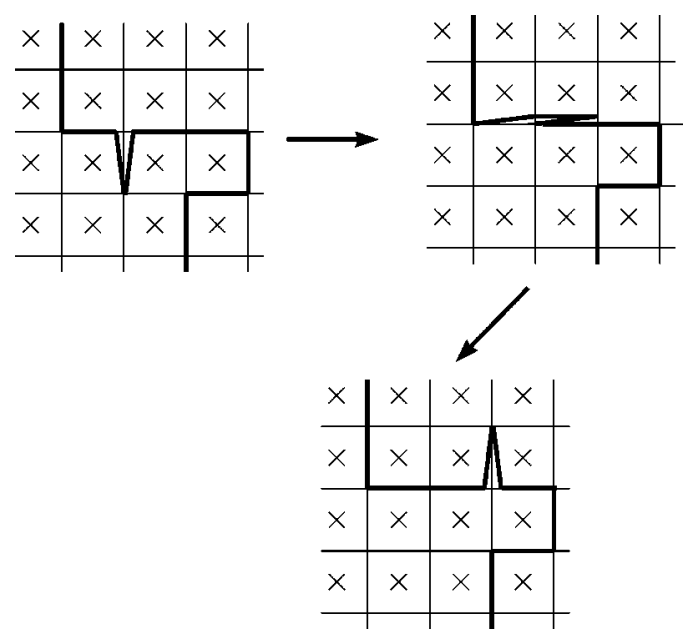

FIG. 1. A realization of reptational dynamics in the EvansEdwards lattice model (two-dimensional illustration). The crosses denote impenetrable obstacles which allow only for "hairpin" moves as shown by the sequence of pictures. The hairpins represent the wiggles of spared length.

the interior of the tube only little wiggles of "spared length" are mobile, as illustrated in Fig. 1 for the special case of a lattice model. These wiggles carry out Brownian motion along the chain. If a wiggle reaches a chain end, it may decay and prolong the tube by its spared length. Chain ends also may produce new wiggles that then diffuse into the interior of the tube. This shortens the tube by the spared length of the newly created wiggle. In the long run, this random motion of the chain ends leads to a complete destruction of the initial tube.

This very simple dynamical model involves several time scales. It needs a microscopic time $T_{0}$ until the segment motion feels the existence of the constraints. $T_{0}$ generally is identified with the Rouse time of a chain of length equal to the entanglement length $N_{e}$,

$$
T_{0} \sim N_{e}^{2} .
$$

$T_{0}$ is relevant for the short-time dynamics of melts, where the tube diameter typically is found to be quite large $[10,11]$, $N_{e} \sim 10-40$. For reptation, $T_{0}$ defines the elementary time step, since this theory does not deal with the unconstrained motion on scale of the tube diameter. A second scale $T_{2}$ is the time a wiggle needs to diffuse over the whole chain. Since in the coarse grained description, the wiggle has to diffuse a distance of $N / N_{e}$ steps, one finds

$$
T_{2} \sim T_{0}\left(\frac{N}{N_{e}}\right)^{2} \sim N^{2} .
$$

$T_{2}$ thus is of the order of the Rouse time of the whole chain. Finally, the reptation time $T_{3}$ is needed to destruct the initial tube completely. Reptation theory [1] predicts

$$
T_{3} \sim T_{0}\left(\frac{N}{N_{e}}\right)^{3}
$$


as limiting result for long chains. Asymptotically, power laws as function of $t$ and $N$ are predicted to hold for the segment motion or the motion of the center of mass in the time windows $T_{0} \ll t \ll T_{2}, T_{2} \ll t \ll T_{3}$, and $T_{3} \ll t$. As a typical result we quote the mean squared spatial displacement of some bead of the chain,

$$
\left\langle\overline{\left[\mathbf{r}_{j}(t)-\mathbf{r}_{j}(0)\right]^{2}}\right\rangle \sim \begin{cases}t^{1 / 4} & \text { for } T_{0} \ll t \ll T_{2} \\ (t / N)^{1 / 2} & \text { for } T_{2} \ll t \ll T_{3} \\ t / N^{2} & \text { for } T_{3} \ll t .\end{cases}
$$

Here $\mathbf{r}_{j}(t)$ gives the spatial position of bead $j$ at time $t$. The bar indicates the (dynamic) average over the stochastic motion and the angular brackets denote the (static) average over all tube configurations.

\section{B. Previous results for the coherent structure function}

We consider a chain of $N+1$ beads ( $N$ segments), labeled by $j=0,1, \ldots, N$. The coherent structure function is defined as

$$
S_{c}(q, t, N)=\sum_{j, k=0}^{N}\left\langle\overline{e^{i \mathbf{q} \cdot\left[\mathbf{r}_{j}(t)-\mathbf{r}_{k}(0)\right]}}\right\rangle .
$$

By definition, $S_{c}(q, t, N)$ refers to a single chain. It can be measured by appropriately labeling a few chains in the system. Reptation results for $S_{c}(q, t, N)$ previously have been derived by Doi and Edwards [13] and by de Gennes [12].

Doi and Edwards have evaluated a simplified version of the reptation model, where the internal motion of the chain is neglected. The physical chain is replaced by a "primitive chain," which only can slide along the tube so that all segments experience the same curvilinear displacement $\Delta \xi(t)$. This model therefore reduces the dynamics to diffusive motion of the single stochastic variable $\Delta \xi$. For the coherent structure function, it yields the result (see Ref. [2], Chap. 6.3.4)

$$
\begin{gathered}
\frac{S_{c}(q, t, N)}{S_{c}(q, 0, N)}=\bar{S}_{D E}\left(q^{2} R_{g}^{2}, \frac{t}{\tau_{d}}\right), \\
\bar{S}_{D E}(Q, \tau)=\frac{Q}{D(Q)} \sum_{p=1}^{\infty} \frac{\sin ^{2} \alpha_{p} e^{-\left(4 / \pi^{2}\right) \alpha_{p}^{2} \tau}}{\alpha_{p}^{2}\left(Q^{2} / 4+Q / 2+\alpha_{p}^{2}\right)},
\end{gathered}
$$

where $R_{g}^{2}$ is the radius of gyration and $\tau_{d} \sim T_{3} \sim N^{3}$. $D(Q)$ is the Debye function,

$$
D(Q)=\frac{2}{Q^{2}}\left(e^{-Q}-1+Q\right) .
$$

The $\alpha_{p}=\alpha_{p}(Q)$ are the positive solutions of

$$
\alpha_{p} \tan \alpha_{p}=\frac{Q}{2} .
$$

Neglecting all internal motions, the result can be applied only for $t \gg T_{2}$, i.e., in a time regime where the internal de- grees of freedom are equilibrated. In the limit of large wave numbers $Q=q^{2} R_{g}^{2} \gg 1$, the result reduces to

$$
\bar{S}_{D E}(Q, \tau)=\frac{8}{\pi^{2}} \sum_{p=1}^{\infty}(2 p-1)^{-2} \exp \left[-(2 p-1)^{2} \tau\right] .
$$

This is the scattering from that part of the primitive chain which at time $t$ still resides in the initial tube $[1,12]$.

The limit $q^{2} R_{g}^{2} \gg 1$ has also been considered by de Gennes. Taking the internal relaxation of the chain into account, his result [12] for the normalized coherent scattering function is a sum of two terms,

$$
\bar{S}_{d G}(q, t, N)=\left[1-B_{d G}(q)\right] \bar{S}^{(l)}(q, t)+B_{d G}(q) \bar{S}^{(c)}(t, N),
$$

$$
B_{d G}(q)=1-\frac{N_{e}}{6 N} q^{2} R_{g}^{2} .
$$

The "creep term" $\bar{S}^{(c)}(t, N)$ is given by Eq. (2.9) and thus describes the large time behavior $t \gg T_{2}$. It tends to 1 for $t / T_{3} \rightarrow 0$. The "local term" $\bar{S}^{(l)}(q, t)$ is taken from an approximate evaluation of the internal relaxation of an infinitely long one-dimensional Rouse chain, folded into the three-dimensional random walk configuration of a tube of $N / N_{e}$ segments. The result reads

$$
\bar{S}^{(l)}(q, t)=e^{t_{1}} \quad \operatorname{erfc} \sqrt{t_{1}},
$$

where

$$
t_{1}=\frac{3}{\pi^{2}} \frac{N}{N_{e}}\left(q^{2} R_{g}^{2}\right)^{2} \frac{t}{\tau_{d}}=\frac{t}{T_{q}} .
$$

This introduces an additional $q$-dependent time scale

$$
T_{q}=\frac{\pi^{2}}{3} \frac{N_{e}}{N} \frac{\tau_{d}}{\left(q^{2} R_{g}^{2}\right)^{2}},
$$

which in view of $R_{g}^{2} \sim N$, and $\tau_{d} \sim N^{3}$ is independent of $N$. $T_{q}$ governs the relaxation of segment density fluctuations on scale $q^{-1}$. In view of $q^{2} R_{g}^{2} \gg 1, T_{q}$ is much smaller than $\tau_{d}$, and for times $t \lesssim T_{q}$, the creep term is constant, $\bar{S}^{(c)}(t, N)$ $\approx \bar{S}^{(c)}(0, N)=1$. On top of this plateau, $\bar{S}^{(l)}(q, t)$ yields a peak rapidly decreasing in time. Note that $\bar{S}^{(l)}(q, t)$ for $t_{1}$ $\gg 1$ behaves as $\bar{S}^{(l)}(q, t)=\left(\pi t_{1}\right)^{-1 / 2}$. The amplitude of the peak is determined by $B_{d G}$, which only depends on $q^{2}$ and $N_{e}$.

Both these approaches neglect end effects such as tube length fluctuations, which are governed by the time scale $T_{2}$. The approximations involved greatly simplify the analysis but are no essential part of the reptation model. In the sequel, we present an analysis of the full model, accounting for the internal degrees of freedom and the finite chain length. Since all the dynamics is driven by the diffusion of the spared length as the only stochastic process, this yields a unified 
description of local relaxation, global creep, and tube length fluctuations. We will find that tube length fluctuations, in particular, have an important influence for intermediate times and chain lengths. Internal relaxation, however, is of much less influence than the results referred to above suggest.

\section{FORMULATION OF THE FULL REPTATION MODEL}

\section{A. Microscopic dynamics}

We here recall the essential features of our model. A more detailed discussion can be found in Ref. [8]. The chain is modeled as a random walk of $N$ steps of fixed length $\mid \mathbf{r}_{j}$ $-\mathbf{r}_{j-1} \mid=l_{0}, j=1, \ldots, N$. The motion is due to the diffusion of wiggles of spared length $l_{s}$. These are represented by particles hopping along the chain from bead to bead, with hopping probability $p$ per time step. The particles do not interact, and a given particle sees the others just as a part of the chain. If a particle passes a bead $j$, it tracks it along by a distance of the spared length $l_{s}$, which is taken to be the same for all particles. The end beads $j=0, N$ of the chain are coupled to large reservoirs that absorb and emit particles at such a rate that the equilibrium density $\rho_{0}$ of particles on the chain is maintained on average. Keeping track of the change of the particle number in these reservoirs, we control the motion of the chain ends: creation or decay of a wiggle at a chain end implies emission or absorption of the corresponding particle by the reservoir.

For the motion of beads in the interior of the tube, the essential stochastic variable of the model is the number $n(j, t)$ of particles which passed over bead $j$ within time interval $[0, t]$ :

$$
n(j, t)=n_{+}(j, t)-n_{-}(j, t) .
$$

Here $n_{ \pm}(j, t)$ is the number of particles that came from the "left" $\left(j^{\prime}<j\right)$ or from the "right" $\left(j^{\prime}>j\right)$, respectively. Consider, for instance, the motion of segment $j$ for a time interval in which it stays in the original tube. Its displacement in the tube is given by $l_{s} n(j, t)$, and since the tube has a random walk configuration, its spatial displacement is given by

$$
\left\langle\overline{\left[r_{j}(t)-r_{j}(0)\right]^{2}}\right\rangle=l_{s} l_{0} \overline{|n(j, t)|} .
$$

Since the underlying stochastic process is single particle hopping, the distribution function of $n(j, t)$ is easily calculated, with the result [Ref. [8], Eq. (3.22)]

$$
\mathcal{P}_{1}(n ; j, t)=\overline{\delta_{n, n(j, t)}}=e^{-\overline{n^{2}(j, t)}} I_{n}\left[\overline{n^{2}(j, t)}\right]
$$

where $I_{n}(z)$ is the modified Bessel function of the first kind. The second moment $\overline{n^{2}(j, t)}$ is found as [see Ref. [8], Eqs. (3.24),(3.12),(3.9)]

$$
\overline{n^{2}(j, t)}=2 \rho_{0} A_{1}(j, t)
$$

$$
A_{1}(j, t)=\frac{p t}{N}+\frac{1}{2 N} \sum_{\kappa=1}^{N-1}\left(1-\alpha_{\kappa}^{t}\right) \frac{\cos ^{2}\left[\frac{\pi \kappa}{N}\left(j+\frac{1}{2}\right)\right]}{\sin ^{2}\left(\frac{\pi \kappa}{2 N}\right)}
$$

$$
\alpha_{\kappa}=1-4 p \sin ^{2} \frac{\pi \kappa}{2 N}
$$

Some useful properties of $A_{1}(j, t)$ are collected in Ref. [8], Appendix A. We also will need the first moment $\overline{|n(j, t)|}$, which from Ref. [8], Eqs. (3.26), (3.27), is found as

$$
\begin{aligned}
\overline{|n(j, t)|}= & \frac{2}{\sqrt{\pi}}\left[\rho_{0} A_{1}(j, t)\right]^{1 / 2}\left\{1-F_{1}\left[4 \rho_{0} A_{1}(j, t)\right]\right\} \\
F_{1}(z)= & \frac{1}{2 \sqrt{\pi}} \int_{0}^{z} d x x^{-3 / 2} e^{-x}\left[\left(1-\frac{x}{z}\right)^{-1 / 2}-1\right] \\
& -\frac{1}{2 \sqrt{\pi}} \Gamma\left(-\frac{1}{2}, z\right)
\end{aligned}
$$

where $\Gamma(y, z)$ is the incomplete $\Gamma$ function.

Except for microscopic times $t \leqslant 2 / p, \alpha_{\kappa}^{t}$ can be approximated as

$$
\alpha_{\kappa}^{t} \approx \exp \left[-4 p t \sin ^{2} \frac{\pi \kappa}{2 N}\right],
$$

so that the theory involves time only in the combination

$$
\hat{t}=p t
$$

In evaluating the theory, we will use $\hat{t}$ as time variable. For $\overline{n^{2}(j, t)} \geqslant 100$, which for $N \gtrsim 100$ implies $\hat{t} \gtrsim 10^{4}, \mathcal{P}_{1}(n ; j, t)$ is well represented by a simple Gaussian

$$
\mathcal{P}_{1}(n ; j, t) \approx\left[2 \pi{\overline{n^{2}(j, t)}}^{-1 / 2} \exp \left(-\frac{n^{2}}{2 \overline{n^{2}(j, t)}}\right)\right.
$$

Knowledge of $\mathcal{P}_{1}(n ; j, t)$ is sufficient as long as we consider motion inside the initial tube. End effects introduce a more complicated quantity. Within time interval $[0, t]$, the tube from the end $j=0$ is destructed up to bead $j<$, where $j<$ is defined as

$$
\begin{gathered}
j_{<}=\bar{l}_{s} n_{\max }(0, t), \\
n_{\max }(0, t)=\max _{s \in[0, t]}[-n(0, s)] .
\end{gathered}
$$

Here

$$
n(0, s)=m_{0}(s)-m_{0}(0),
$$


where $m_{0}(s)$ is the occupation number at time $s$ of the reservoir at chain end 0 . Thus $n_{\max }(0, t)$ is the maximal negative fluctuation of the occupation number of reservoir $j=0$ in the time interval $[0, t]$. In Eq. (3.12) we also introduced

$$
\bar{l}_{s}=l_{s} / l_{0},
$$

measuring all lengths in units of the segment size $l_{0}$. Similarly, from the other end tube destruction within time $t$ proceeds to bead

$$
j_{>}=N-\bar{l}_{s} n_{\max }(N, t),
$$

with $n_{\max }(N, t)$ being the maximal negative fluctuation of the occupation number $m_{N}(s)$ of the reservoir at chain end $N$. The stochastic processes $m_{0}(s)$ or $m_{N}(s)$ are not Markovian, since a particle emitted by a reservoir can be reabsorbed by the same reservoir later. This induces a correlation that dies out only if the particle has time to reach the other reservoir, i.e., on time scale $T_{2}$. For such a correlated process, the distribution and the moments of $n_{\max }$ cannot be calculated rigorously, even though arbitrary moments of $n(0, s)$, involving any number of time variables $s$, can be evaluated (see Ref. [8], Sec. III). As soon as tube renewal comes into play, we therefore have to resort to some approximation.

Some important quantity entering our theory is the average $\overline{n_{\max }(0, t)}$. It, for instance, yields the motion of the end segment via the relation [Ref. [8], Eq. (2.12)]

$$
\left\langle\overline{\left[\mathbf{r}_{0}(t)-\mathbf{r}_{0}(0)\right]^{2}}\right\rangle=2 \bar{l}_{S} l_{0}^{2} \overline{n_{\max }(0, t)} .
$$

We use the expression [Ref. [8], Eq. (5.1)]

$$
\overline{n_{\max }(0, t)}=\sum_{s=1}^{t} \overline{\frac{|n(0, s)|}{2 s},}
$$

which is correct for a Markov process. Using in Eq. (3.16) the exact moments $\overline{|n(0, s)|}$ [Eq. (3.7)], we in essence approximate the correlated process by a sequence of Markov processes which for each time step $s$ yield the correct instantaneous value of $\overline{|n(0, s)|}$. This "mean hopping rate" approximation, which was discussed in more detail in Ref. [8], gives good results for larger times. For microscopic times, it underestimates $\overline{n_{\max }(0, t)}$ by about a factor of 2 , but with increasing time it approaches the full result for $\overline{n_{\max }}$ as found in simulations. For $t \approx T_{2}$, the deviation for the motion of the end segment, which is most sensitive to our approximation, is of the order of $10 \%$ only (see Fig. 9 of Ref. [9]).

\section{B. Outline of our calculation of the coherent structure function}

The basic quantity to be considered is the scattering from a pair of beads

$$
S(q, t ; j, k, N)=\left\langle\overline{e^{i \mathbf{q} \cdot\left[\mathbf{r}_{j}(t)-\mathbf{r}_{k}(0)\right]}}\right\rangle,
$$

which must be summed over the beads to find the coherent structure function $S_{c}(q, t ; N)$. To get some information on the contribution of the different parts of the chain, we consider a slight generalization in which we sum only over the $M+1$ central beads

$$
S_{c}(q, t ; M, N)=\sum_{j, k=(N-M) / 2}^{(N+M) / 2} S(q, t ; j, k, N) .
$$

Clearly, the coherent structure function of the full chain is

$$
S_{c}(q, t ; N)=S_{c}(q, t ; N, N)
$$

To calculate $S(q, t ; j, k, N)$, we first consider the contribution $S^{(T)}(q, t ; j, k, N)$, which results from those stochastic motions for which a part of the initial tube still exists at time $t$. [The upper index $(T)$ stands for "tube."] We then can set up an integral equation for $S(q, t ; j, k, N)$, in which $S^{(T)}(q, t ; j, k, N)$ shows up as inhomogeneity (see Sec. VI). Furthermore, for $t \ll T_{3}$, contributions where the tube is destroyed completely, are negligible, and $S^{(T)}$ coincides with $S$.

$S^{(T)}(q, t ; j, k, N)$ incorporates the effects of internal relaxation and tube length fluctuations, and its calculation is the most tedious part of our analysis. We here need to simultaneously control the motion of segment $j$ and of the chain ends. More specifically, we will need the distribution function

$$
\mathcal{P}_{\text {max }, j}^{(T)}\left(n_{m}, n_{j} ; t\right)=\overline{\Theta\left(j_{<}-j_{>}\right) \delta_{n_{m}, n_{\text {max }}(0, t)} \delta_{n_{j}, n(j, t)}},
$$

i.e., the simultaneous distribution of $n(j, t)$ and $n_{\max }(0, t)$ under the constraint that a part of the initial tube still exists. Again the correlated nature of the stochastic motion of the chain ends prevents a rigorous evaluation of $\mathcal{P}_{\text {max }, j}^{(T)}$, and we use random walk theory to construct an approximate functional form. The result depends on $n_{m}, n_{j}$, and $t$ only through the rescaled variables $n_{m} / \overline{n_{m}(t)}$ and $n_{j} / \overline{\left[n_{j}^{2}(t)\right]^{1 / 2}}$, and in the spirit of our mean hopping rate approximation, we in these variables replace the normalizing factors $\overline{n_{m}(t)}$ and ${\overline{\left[n_{j}^{2}(t)\right.}}^{1 / 2}$ of the random walk by their counterparts for the proper correlated process. In essence, this again amounts to replacing the correlated stochastic motion of the chain ends by a whole sequence of uncorrelated random walks, parametrized by an effective hopping rate $p^{\prime}$. This hopping rate is adjusted such that the random walk that replaces the correlated process for final time $t$, at that time yields the correct moments $\overline{n_{m}(t)}=\overline{n_{\max }(0, t)}$ and $\overline{n_{j}^{2}(t)}=\overline{[n(j, t)]^{2}}$. [It in fact yields the correct Gaussian distribution of the single variable $n(j, t)$.] As discussed in Ref. [8], Sec. V B, $p^{\prime}$ changes from a value $\rho_{0} p$ at microscopic times to $\rho_{0} p / N$ for $t \gg T_{2}$. Since $\rho_{0} p$ governs the short time motion of a segment whereas $\rho_{0} p / N$ is the mobility of the primitive chain, the mean hopping rate approximation smoothly interpolates between these more rigorously accessible limits. This will be discussed again in Sec. IV C, after we present the details of our approach.

Our theory involves three important time (and segment index) dependent parameter functions, 


$$
c=c(t)=\sqrt{\frac{\pi}{2}} \bar{l}_{s} \overline{n_{\max }(0, t)}
$$

measures the extent of tube destruction and thus accounts for tube length fluctuations. For $\overline{n_{\max }(0, t)}$, we use the approximation (3.16). It turns out that the time dependence of $c(t)$, which very slowly tends to its asymptotic limit $c(t)$ $\stackrel{t \rightarrow \infty}{\rightarrow}$ const $t^{1 / 2}$ [cf. Eq. (7.2)], is responsible for the well

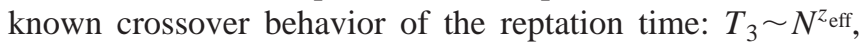
where $z_{\text {eff }}$ slowly approaches its asymptotic value $z_{\text {eff }} \longrightarrow 3$ from above. (A detailed discussion of the reptation time will be given in a separate paper.)

A second function, $a(j, t)$, measures the coupling of the motion of an interior segment $j$ to the motion of a chain end. Initially, this coupling vanishes, but it increases with time due to particles created at a chain end and traveling over segment $j$. If this coupling is fully developed, all segments approximately have moved the same distance in the tube and the primitive chain model results. The precise definition of $a(j, t)$ is given in Eq. (4.17).

Finally, it should be noted that the effective mobility of a segment for $t \ll T_{2}$ depends on its position in the chain, an effect already present for free Rouse type motion. This is taken into account by the function $b(j, t)$, which is defined in Eq. (4.36).

Having described the main ideas of our approach, we now turn to the details. We first construct and analyze the tube conserving contribution $S_{c}^{(T)}(q, t ; M, N)$ to the structure function.

\section{TUBE CONSERVING CONTRIBUTION TO THE STRUCTURE FUNCTION}

In this and the following section, we consider the contribution of those stochastic processes, which do not destroy the initial tube completely, i.e., we insist on the inequality

$$
j_{>}-j_{<} \geqslant 0,
$$

since due to the definitions (3.12),(3.15), the tube has been destroyed up to segment $j_{<}$from chain end 0 or $j>$ from chain end $N$, respectively. We first construct a formally exact expression for the corresponding contribution $S^{(T)}(q, t ; j, k, N)$. Its summation over indices $j$ and $k$ as in Eqs. (3.18) and (3.19) yields the tube conserving contribution to the coherent structure function.

\section{A. Exact expression for $S^{(T)}(q, t ; j, k, N)$}

Depending on the relation among $j, k, j_{<}$, and $j_{>}$, we have to distinguish several cases. We use the notation

$$
j(t)=j+\bar{l}_{s} n(j, t)
$$

and we illustrate the analysis with two typical cases shown in Fig. 2. Figure 2 a gives a schematic sketch of a situation, in which the inequalities

$$
j(t) \leqslant k, \quad j_{<} \leqslant k, \quad j_{>} \geqslant j(t),
$$

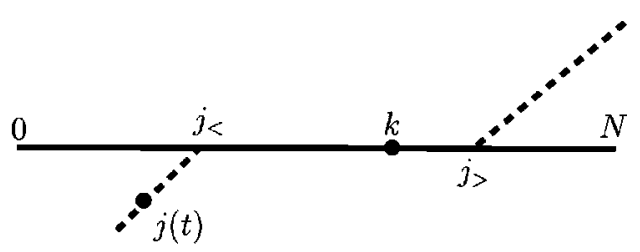

a)

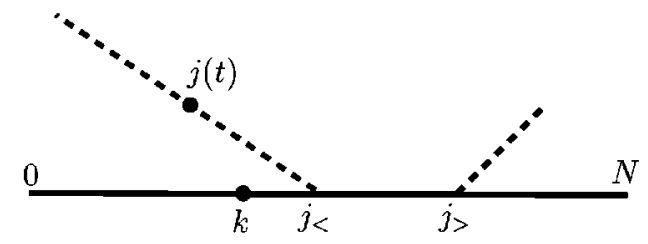

b)

FIG. 2. Schematic drawings of the cases discussed in the text. The full line represents the unfolded initial tube. Broken lines represent the new end pieces of the unfolded tube created up to time $t$.

hold. That means that tube renewal from chain end 0 has not passed over the original position of segment $k$, and segment $j$ at time $t$ is not found in the part of the new tube created from chain end $N$. Furthermore, the new position of segment $j$, if measured along the tube, is closer to the new position of chain end zero than the original position of segment $k$. The relative ordering of $k$ and $j_{>}$, or of $j(t)$ and $j_{<}$is unimportant. As is clear from Fig. 2(a), the path connecting $j(t)$ and $k$ has $k-j(t)$ steps, and since the chain configuration is a random walk, we find

$$
\left\langle e^{i \mathbf{q} \cdot\left[\mathbf{r}_{j}(t)-\mathbf{r}_{k}(0)\right]}\right\rangle=e^{-\bar{q}^{2}[k-j(t)]},
$$

where

$$
\bar{q}^{2}=\frac{q^{2} l_{0}^{2}}{6}
$$

As a result, the contribution of such configurations to $S^{(T)}(q, t ; j, k, N)$ reads

$$
\overline{\Theta(k-j(t)) \Theta\left(k-j_{<}\right) \Theta\left(j_{>}-j(t)\right) \Theta\left(j_{>}-j_{<}\right) e^{-\bar{q}^{2}[k-j(t)]}},
$$

where the discrete $\Theta$ function is defined as

$$
\Theta(n)= \begin{cases}1, & n \equiv 0,1,2, \ldots \\ 0, & n=-1,-2, \ldots .\end{cases}
$$

Now consider a typical case of other type, shown in Fig. 2(b). It is defined by the inequalities

$$
j(t) \leqslant k, \quad j<>k,
$$

and differs from the previous one in that tube renewal from chain end 0 has passed over the original position of segment 
$k$. The thus created part of the new tube necessarily contains the new position of segment $j$, and the random walk connecting $j(t)$ to $k$ has $\left(j_{<}-k\right)+\left(j_{<}-j(t)\right)$ steps. We thus find the contribution

$$
\overline{\Theta(k-j(t)) \Theta\left(j_{<}-k-1\right) \Theta\left(j_{>}-j_{<}\right) e^{-\bar{q}^{2}\left[2 j_{<}-k-j(t)\right]}} .
$$

The other cases compatible with $j_{>} \geqslant j_{<}$are given by the relations $\left[j(t) \leqslant k, j_{>}<j(t)\right], \quad\left[j(t)>k, j_{<} \leqslant j(t), j_{>} \geqslant k\right]$, $[j(t)>k, j<>j(t)]$, and $\left[j(t)>k, j_{>}<k\right]$. Proceeding as above, after some manipulations with the $\Theta$ functions, we arrive at the result

$$
\begin{aligned}
S^{(T)}(q, t ; j, k, N)= & \mathcal{S}_{1}(q, t ; j, k, N)+\mathcal{S}_{2}(q, t ; j, k, N) \\
& +\mathcal{S}_{3}(q, t ; j, k, N),
\end{aligned}
$$

where

$$
\mathcal{S}_{1}(q, t ; j, k, N)=\overline{e^{-\bar{q}^{2}|k-j(t)|}}
$$

is the contribution ignoring $j_{<, j_{>}}$, thus ignoring all end effects. $\mathcal{S}_{2}$ corrects $\mathcal{S}_{1}$ for the constraint $j_{>} \geqslant j_{<}$,

$$
\mathcal{S}_{2}(q, t ; j, k, N)=\overline{\left[\Theta\left(j_{>}-j_{<}\right)-1\right] e^{-\bar{q}^{2}|k-j(t)|}},
$$

and $\mathcal{S}_{3}$ takes the newly created parts of the tube into account

$$
\begin{aligned}
\mathcal{S}_{3}(q, t ; j, k, N)= & \overline{\Theta(k-j(t)) \Theta\left(j_{<}-k-1\right) \Theta\left(j_{>}-j_{<}\right)\left[e^{-\bar{q}^{2}\left[2 j_{<}-k-j(t)\right]}-e^{-\bar{q}^{2}[k-j(t)]}\right]} \\
& +\overline{\Theta(j(t)-k-1) \Theta\left(j_{<}-j(t)-1\right) \Theta\left(j_{>}-j_{<}\right)\left[e^{-\bar{q}^{2}\left[2 j_{<}-k-j(t)\right]}-e^{-\bar{q}^{2}[j(t)-k]}\right]} \\
& +\overline{\Theta(k-j(t)) \Theta\left(j(t)-j_{>}-1\right) \Theta\left(j_{>}-j_{<}\right)\left[e^{-\bar{q}^{2}\left[k+j(t)-2 j_{>}\right]}-e^{-\bar{q}^{2}[k-j(t)]}\right]} \\
& +\overline{\Theta(j(t)-k-1) \Theta\left(k-j_{>}-1\right) \Theta\left(j_{>}-j_{<}\right)\left[e^{-\bar{q}^{2}\left[k+j(t)-2 j_{>}\right]}-e^{-\bar{q}^{2}[j(t)-k]}\right]}
\end{aligned}
$$

These expressions are formally exact, but, as pointed out in the preceding section, to evaluate $\mathcal{S}_{2}$ and $\mathcal{S}_{3}$, we have to construct an approximation for the simultaneous distribution of $n(j, t), j_{<}$, and $j_{>} \cdot \mathcal{S}_{1}$ could be evaluated with the exactly known distribution of $n(j, t)$ [Eq. (3.3)]. However, being interested in the universal features of the model, which only show up for larger times, we use the Gaussian approximation (3.11) and ignore the discreteness of the elementary hopping process. We also will take the chain as continuous, in the evaluation replacing segment summations by integrals. A priori these simplifications might influence the short time behavior, but in practice they are found to have no measurable effects. For a check, we numerically have compared the continuous model to a fully discrete evaluation. For the properly normalized coherent structure function $S_{c}(q, t ; M, N) / S_{c}(q, 0 ; M, N)$, the difference for all times, including the microscopic range, is found to be of the order $10^{-3}$ and thus negligible.

\section{B. The contribution $\mathcal{S}_{1}(q, t ; j, k, N)$}

Combining Eqs. (4.2) and (4.6) with the definition (3.3) of $\mathcal{P}_{1}$, we find

$$
\mathcal{S}_{1}(q, t ; j, k, N)=\sum_{n=-\infty}^{+\infty} e^{-\bar{q}^{2}\left|k-j-\bar{l}_{s} n\right|} \mathcal{P}_{1}(n ; j, t)
$$

With the Gaussian approximation (3.11) for $\mathcal{P}_{1}(n, j, t)$ and with $n$ taken continuous, this expression is easily evaluated to yield

$$
\begin{aligned}
\mathcal{S}_{1}(q, t ; j, k, N)= & \frac{1}{2} e^{Q^{2}}\left[e^{2 \Delta Q} \operatorname{erfc}(Q+\Delta)\right. \\
& \left.+e^{-2 \Delta Q} \operatorname{erfc}(Q-\Delta)\right],
\end{aligned}
$$

where

$$
\begin{aligned}
& Q=\bar{q}^{2} \bar{l}_{s} \sqrt{\rho_{0} A_{1}(j, t)}, \\
& \Delta=\frac{k-j}{2 \bar{l}_{s} \sqrt{\rho_{0} A_{1}(j, t)}} .
\end{aligned}
$$

Note that Eqs. (3.2) and (3.7) imply

$$
\sqrt{\rho_{0} A_{1}(j, t)} \sim\left\langle\overline{\left[r_{j}(t)-r_{j}(0)\right]^{2}}\right\rangle,
$$

so that in the result (4.10), $q$ and $k-j$ are measured relative to the mean displacement of segment $j$. Even though the Gaussian approximation from its derivation holds only for $\rho_{0} A_{1} \gg 1$, it for $\rho_{0} A_{1} \rightarrow 0$, i.e., $t \rightarrow 0$, reproduces the exact static behavior of our model,

$$
\mathcal{S}_{1}(q, 0 ; j, k, N)=e^{-\bar{q}^{2}|k-j|}
$$

As will be discussed in Sec. VE, this is an important requirement for any theory of the dynamic scattering functions.

\section{Distribution function for $\mathcal{S}_{3}(q, t ; j, k, N)$}

In view of the symmetry of the chain under reflection $j$ $\rightarrow N-j$, the last two terms in Eq. (4.8) for $\mathcal{S}_{3}$, when summed 
over $j$ and $k$, yield contributions identical to the first two terms. We therefore can restrict the analysis to the first terms, which involve the distribution function referred to in Sec. III B [Eq. (3.20)] :

$$
\mathcal{P}_{\max , j}^{(T)}\left(n_{m}, n_{j} ; t\right)=\overline{\Theta\left(j_{>}-j_{<}\right) \delta_{n_{m}, n_{\max }(0, t)} \delta_{n_{j}, n(j, t)}} .
$$

For instance, in terms of this distribution function, the contribution to the coherent scattering function $S_{c}(q, t, N)$ of the first term in Eq. (4.8) reads

$$
\begin{aligned}
\sum_{j, k} & \sum_{n_{m}, n_{j}} \Theta\left(k-j-\bar{l}_{s} n_{j}\right) \Theta\left(\bar{l}_{s} n_{m}-k-1\right) \mathcal{P}_{\max , j}^{(T)}\left(n_{m}, n_{j} ; t\right) \\
& \times\left[e^{-\bar{q}^{2}\left(2 \bar{l}_{s} n_{m}-k-j-\bar{l}_{s} n_{j}\right)}-e^{-\bar{q}^{2}\left(k-j-\bar{l}_{s} n_{j}\right)}\right] .
\end{aligned}
$$

We now construct an approximate expression for $\mathcal{P}_{\max , j}^{(T)}$, based on random walk theory. We first present the essential steps of our approach and discuss the approximations involved thereafter. Some details of the calculations are given in Appendix A.

We introduce the auxiliary variable $n_{0}=-n(0, t)$ and write

$$
\begin{aligned}
\mathcal{P}_{\max , j}^{(T)}\left(n_{m}, n_{j} ; t\right) \\
=\sum_{n_{0}=-\infty}^{+\infty} \overline{\Theta(j>-j<) \delta_{n_{m}, n_{\max }(0, t)} \delta_{n_{j}, n(j, t)} \delta_{n_{0},-n(0, t)}} \\
=\sum_{n_{0}=-\infty}^{+\infty} \mathcal{P}_{\max , 0, j}^{(T)}\left(n_{m}, n_{0}, n_{j} ; t\right) .
\end{aligned}
$$

Recall that $n(0, t)$ is the change in the occupation of reservoir 0 within time $t$. We then factorize according to

$$
\mathcal{P}_{\max , 0, j}^{(T)}\left(n_{m}, n_{0}, n_{j} ; t\right) \rightarrow \frac{\mathcal{P}_{\max , 0}^{(T)}\left(n_{m}, n_{0} ; t\right) \mathcal{P}_{2}\left(n_{0}, n_{j} ; 0, j, t\right)}{\mathcal{P}_{1}\left(n_{0} ; 0, t\right)} .
$$

Here $\mathcal{P}_{\max , 0}^{(T)}\left(n_{m}, n_{0} ; t\right)$ is the simultaneous distribution of $n_{\text {max }}(0, t)$ and $-n(0, t)$, with the constraint $j_{>} \geqslant j_{<}$taken into account. $\mathcal{P}_{2}\left(n_{0}, n_{j} ; 0, j, t\right)$ is the simultaneous distribution of $-n(0, t)$ and $n(j, t)$, so that $\mathcal{P}_{2}\left(n_{0}, n_{j} ; 0, j, t\right) / \mathcal{P}_{1}\left(n_{0} ; 0, t\right)$ is the conditional probability to find $n_{j}=n(j, t)$, once $n_{0}$ $=-n(0, t)$ is given. A rigorous expression for $\mathcal{P}_{2}$ was given in Ref. [8], Sec. III. Here we again use the Gaussian approximation [Ref. [8], Appendix C, Eq. (C7)]. Using also the Gaussian approximation (3.11) for $\mathcal{P}_{1}\left(n_{0} ; 0, t\right)$, we find

$$
\begin{aligned}
& \frac{\mathcal{P}_{2}\left(n_{0}, n_{j} ; 0, j, t\right)}{\mathcal{P}_{1}\left(n_{0} ; 0, t\right)} \\
& =\left[2 \pi \overline{n^{2}(j, t)}\right]^{-1 / 2}\left(1-a^{2}\right)^{-1 / 2} \\
& \quad \times \exp \left[-\frac{1}{2\left(1-a^{2}\right)}\left(a^{2} z_{0}^{2}+z_{j}^{2}-2 a z_{0} z_{j}\right)\right],
\end{aligned}
$$

where

$$
z_{j}=\frac{n_{j}}{\sqrt{\overline{n^{2}(j, t)}}}, \quad z_{0}=\frac{n_{0}}{\sqrt{\overline{n^{2}(0, t)}}},
$$

$$
a=a(j, t)=\frac{\widetilde{A}_{3}(j, t)}{\left[A_{1}(0, t) A_{1}(j, t)\right]^{1 / 2}},
$$

with [cf. Ref. [8], Eq. (A12)]

$$
\begin{aligned}
\widetilde{A}_{3}(j, t)= & \frac{p t}{N}+\frac{N}{3}-\frac{1}{2}+\frac{1}{6 N}+\frac{j^{2}}{2 N}-\left(1-\frac{1}{2 N}\right) j \\
& -\frac{1}{2 N} \sum_{\kappa=1}^{N-1} \frac{\cos \left(\frac{\pi \kappa}{2 N}\right) \cos \left[\frac{\pi \kappa}{N}\left(j+\frac{1}{2}\right)\right]}{\sin ^{2}\left(\frac{\pi \kappa}{2 N}\right)} \alpha_{\kappa}^{t} .
\end{aligned}
$$

$A_{1}$ and $\alpha_{\kappa}$ are given in Eqs. (3.5) and (3.6), respectively.

This introduces the parameter function $a=a(j, t)$. It measures the coupling of the motion of bead $j$ to the motion of chain end 0 . If it vanishes, the conditional probability (4.15) reduces to $\mathcal{P}_{1}\left(n_{j} ; j, t\right)$. This happens for $p t \ll j^{2}$ [see Ref. [8], Eq. (A16)]. The maximal value of $a$ is 1 , which is approached for $j \rightarrow 0$ and all $t$, or for $t \gg T_{2}$ and all $j$. In the latter limit, Eq. (4.15) yields $z_{j}=z_{0}$, and the motion of all segments is rigidly coupled to the motion of the end segment. In this limit, we thus recover the basic assumption of the primitive chain model.

To find an acceptable functional form for $\mathcal{P}_{\text {max }, 0}^{(T)}\left(n_{m}, n_{0} ; t\right)$, we replace the correlated stochastic process $n(0, s)$ by a random walk $n^{\prime}(s)$ on the integer numbers, with hopping rate $p^{\prime}$. We have to consider walks that start at $n^{\prime}(0)=0$, end at $n^{\prime}(t)=n_{0}$, and attain the maximal value $n_{m} \geqslant n_{0}$ for some $s \in[0, t]$. To take care of the constraint

$$
\frac{1}{\bar{l}_{s}}\left(j_{>}-j_{<}\right)=N^{\prime}-n_{\max }(N, t)-n_{\max }(0, t) \geqslant 0,
$$

we restrict the walk $n^{\prime}(t)$ to the interval $\left[n_{m}-N^{\prime}+1, n_{m}\right]$, where $N^{\prime}$ is the greatest integer less than $N / \bar{l}_{s}$, and we use absorbing boundary conditions. This amounts to the assumption that a particle entering the chain from the reservoir at chain end $N$ is transfered immediately to the reservoir at chain end 0 . This assumption is in the spirit of the primitive chain model.

With these simplifications, $\mathcal{P}_{\max , 0}^{(T)}$ can be calculated as sketched in Appendix A. Our result reads 


$$
\begin{aligned}
\mathcal{P}_{\max , 0}^{(T)}\left(n_{m}, n_{0} ; t\right) & \\
= & \Theta\left(n_{m}-\frac{\left|n_{0}\right|+n_{0}}{2}\right) \Theta\left(N^{\prime}-n_{m}-\frac{\left|n_{0}\right|-n_{0}}{2}\right) \frac{1}{\sqrt{\pi} p^{\prime} t} \\
& \times \sum_{\nu=-\infty}^{+\infty}\left\{(\nu+1)\left(\frac{\nu N^{\prime}}{\sqrt{p^{\prime} t}}+\frac{n_{m}}{\sqrt{p^{\prime} t}}-\frac{n_{0}}{2 \sqrt{p^{\prime} t}}\right)\right. \\
& \times \exp \left[-\left(\frac{\nu N^{\prime}}{\sqrt{p^{\prime} t}}+\frac{n_{m}}{\sqrt{p^{\prime} t}}-\frac{n_{0}}{2 \sqrt{p^{\prime} t}}\right)^{2}\right]-\nu\left(\frac{\nu N^{\prime}}{\sqrt{p^{\prime} t}}\right. \\
& \left.\left.+\frac{n_{0}}{2 \sqrt{p^{\prime} t}}\right) \exp \left[-\left(\frac{\nu N^{\prime}}{\sqrt{p^{\prime} t}}+\frac{n_{0}}{2 \sqrt{p^{\prime} t}}\right)^{2}\right]\right\} .
\end{aligned}
$$

It is valid for $p^{\prime} t \gg 1$ and $N^{\prime} \gg 1$, which is the region of interest here. We now note that for $p^{\prime} t \gg 1$, the relations

$$
\begin{gathered}
\overline{n^{\prime 2}(t)}=2 p^{\prime} t, \\
\bar{n}_{m}=\max _{s \in[0, t]} n^{\prime}(s)=2 \sqrt{\frac{p^{\prime} t}{\pi}}
\end{gathered}
$$

hold, and we use these relations to eliminate the factors $\sqrt{p^{\prime} t}$,

$$
\begin{aligned}
& \frac{n_{0}}{\sqrt{p^{\prime} t}}=\sqrt{2} \frac{n_{0}}{\sqrt{\overline{n_{0}^{2}(t)}}}=\sqrt{2} z_{0}, \\
& \frac{n_{m}}{\sqrt{p^{\prime} t}}=\sqrt{2} \sqrt{\frac{2}{\pi} \frac{n_{m}}{\bar{n}_{m}}}=\sqrt{2} y, \\
& \frac{N^{\prime}}{\sqrt{p^{\prime} t}}=\sqrt{2} \sqrt{\frac{2}{\pi}} \frac{N}{\bar{l}_{s} \bar{n}_{m}}=\sqrt{2} \hat{N} .
\end{aligned}
$$

In the last line, we used $\bar{n}_{m}$, since $N^{\prime}$ is introduced via the constraint (4.19). Furthermore, we write the prefactor in Eq. (4.20) as

$$
\frac{1}{\sqrt{\pi} p^{\prime} t}=\frac{2}{\sqrt{\pi}}\left[\overline{n^{\prime 2}(t)}\right]^{-1 / 2}\left(\sqrt{\frac{\pi}{2}} \bar{n}_{m}\right)^{-1}
$$

and we again treat $z_{0}$ and $y$ as continuous variables, which for $p^{\prime} t \gg 1$ is a valid approximation consistent with our derivation. With these substitutions, Eq. (4.20) reads

$$
\begin{aligned}
d n_{0} d n_{m} & \mathcal{P}_{\max , 0}^{(T)}\left(n_{m}, n_{0} ; t\right) \\
= & \Theta\left(y-\frac{\left|z_{0}\right|+z_{0}}{2}\right) \Theta\left(\hat{N}-y-\frac{\left|z_{0}\right|-z_{0}}{2}\right) \\
& \times \sqrt{\frac{2}{\pi}} d z_{0} d y \sum_{\nu=-\infty}^{+\infty}\left\{(\nu+1)\left(2 \nu \hat{N}+2 y-z_{0}\right)\right. \\
& \times \exp \left[-\frac{\left(2 \nu \hat{N}+2 y-z_{0}\right)^{2}}{2}\right]-\nu\left(2 \nu \hat{N}+z_{0}\right) \\
& \left.\times \exp \left[-\frac{\left(2 \nu \hat{N}+z_{0}\right)^{2}}{2}\right]\right\} .
\end{aligned}
$$

We use this result that has been derived for a random walk, also for the correlated process $n(0, s)$ by reinterpreting the variables. $z_{0}$ is given by Eq. (4.16), and

$$
y=\sqrt{\frac{2}{\pi}} \frac{n_{\max }(0, t)}{n_{\max }(0)},
$$

$$
\hat{N}=N / c,
$$

with

$$
c=c(t)=\sqrt{\frac{\pi}{2}} \bar{l}_{s} \overline{n_{\max }(0, t)} .
$$

For $\overline{n_{\max }(0, t)}$, the approximation (3.16) is used. Up to the factor $\sqrt{\pi / 2}$, the parameter $c$ gives the distance up to which the tube has been destroyed on average. We now use expressions (4.22), (4.15), and (4.14) to evaluate Eq. (4.13), where the sum over $n_{0}$ has to be replaced by the integral over $z_{0}$. Some exercise in Gaussian integrals yields our final result,

$$
\begin{aligned}
d n_{j} d n_{m} \underset{\max , j}{(T)}\left(n_{m}, n_{j} ; t\right)= & \frac{d z_{j} d y}{\sqrt{2 \pi}} \Theta(y) \Theta(\hat{N}-y) \\
& \times \sum_{\nu=-\infty}^{+\infty} \mathcal{P}_{\max , j}^{(\nu)}\left(y, z_{j}, a\right),
\end{aligned}
$$




$$
\begin{aligned}
\mathcal{P}_{\max , j}^{(\nu)}(y, z, a)= & (\nu+1) \exp \left[-\frac{(2 a \nu \hat{N}+2 a y-z)^{2}}{2}\right]\left\{a ( 2 a \nu \hat { N } + 2 a y - z ) \left[\operatorname{erfc}\left(\frac{a z-y+a_{2} y+a_{2} \nu \hat{N}}{\sqrt{a_{2}}}\right)\right.\right. \\
& \left.-\operatorname{erfc}\left(\frac{a z-y+a_{2} y+a_{2} \nu \hat{N}+\hat{N}}{\sqrt{a_{2}}}\right)\right]+\sqrt{\frac{a_{2}}{\pi}}\left[\exp \left(-\frac{\left(a z-y+a_{2} y+a_{2} \nu \hat{N}\right)^{2}}{a_{2}}\right)\right. \\
& \left.\left.-\exp \left(-\frac{\left(a z-y+a_{2} y+a_{2} \nu \hat{N}+\hat{N}\right)^{2}}{a_{2}}\right)\right]\right\}-\nu \exp \left[-\frac{(2 a \nu \hat{N}-z)^{2}}{2}\right]\left\{a ( 2 a \nu \hat { N } - z ) \left[\operatorname{erfc}\left(\frac{a z-y+a_{2} \nu \hat{N}}{\sqrt{a_{2}}}\right)\right.\right. \\
& \left.\left.-\operatorname{erfc}\left(\frac{a z-y+a_{2} \nu \hat{N}+\hat{N}}{\sqrt{a_{2}}}\right)\right]+\sqrt{\frac{a_{2}}{\pi}}\left[\exp \left(-\frac{\left(a z-y+a_{2} \nu \hat{N}\right)^{2}}{a_{2}}\right)-\exp \left(-\frac{\left(a z-y+a_{2} \nu \hat{N}+\hat{N}\right)^{2}}{a_{2}}\right)\right]\right\}
\end{aligned}
$$

with the notation $a_{2}=2\left(1-a^{2}\right)$.

Clearly, the approximations inherent in our construction of $\mathcal{P}_{\max , j}^{(T)}$ need some justification. Steps like the replacement of discrete by continuous variables are well justified, since we need the result only in a time and chain-length regime where a continuous chain model is valid. The problematic steps are the factorization (4.14) of $\mathcal{P}_{\max , 0, j}^{(T)}$ and the calculation of the functional form of $\mathcal{P}_{\max , 0}^{(T)}$ by random walk theory.

Technically, the factorization (4.14) serves to reduce the problem to the treatment of the single stochastic process $n(0, s)$. It clearly is justified for large times, $t \gg T_{2}$, where $n(j, t)$ is firmly bound to $n(0, t)$, and where $\mathcal{P}_{\max , j}^{(T)}$ becomes equivalent to $\mathcal{P}_{\text {max }, 0}^{(T)}$. For shorter times $t \leqq T_{2}$, it assumes that $-n(0, t)$ is a good measure of $n_{\max }(0, t)$, which is certainly incorrect, in particular for $t \ll T_{2}$. However, for $t \ll T_{2}$, end effects influence only a small part of the chain. As can be seen from Eqs. (3.16) and (3.7), and has been explicitly worked out in Ref. [8], Eq. (5.28), $\overline{n_{\max }(0, t)}$ for $t \ll T_{2}$ behaves as $\overline{n_{\max }(0, t)} \sim(p t)^{1 / 4} \sim N^{1 / 2}\left(t / T_{2}\right)^{1 / 4} \ll N$. Since the coherent scattering function sums over all segments, it for such times is dominated by the motion of interior segments not influenced by end effects and governed by the distribution function $\mathcal{P}_{1}(n ; j, t)$. It is easily verified that in the appropriate limit $\hat{N}=N / c(t) \rightarrow \infty$, the distribution function (4.26), when integrated over $n_{m}$, reduces to the Gaussian approximation for $\mathcal{P}_{1}(n ; j, t)$. [Note that in this limit only a part of the $\nu$ $=0$ contribution to the sum in Eq. (4.26) survives.]

We now turn to our construction of $\mathcal{P}_{\text {max }, 0}^{(T)}\left(n_{m}, n_{0}\right)$. Our treatment of the constraint (4.19) should be adequate, since this constraint is relevant only for times of the order of the reptation time, $t \approx T_{3}$, where the internal degrees of freedom of the chain are irrelevant. Furthermore, $\mathcal{P}_{\max , 0}^{(T)}$ by construction obeys the constraint $n_{0} \leqslant n_{m}$. Also, taking $\hat{N} \gg 1$ and integrating over $n_{0}$, we find a distribution with the desired first moment $\overline{n_{m}}=\overline{n_{\max }(0, t)}$. Integrating over $n_{m}$, we find the correct (Gaussian) distribution of $n_{0}$. With these three important features guaranteed, we may hope that we have found a good approximation for the distribution function of the full correlated process.
To summarize, our construction interpolates among two limits where the full dynamics reduces to that of a single stochastic variable. For $t \ll T_{2}$, the motion of individual segments governed by $n(j, t)$ is essential. For $t \gg T_{2}$, the parameter function $a$ tends to 1 and the internal motion becomes irrelevant. Furthermore, the correlations of the stochastic process $n(0, s)$ have died out. We thus are concerned with a single uncorrelated process $n(0, s)$, as in the primitive chain model. Smoothly interpolating among these limits, we may hope to have found a good approximation also in the crossover region $t \approx T_{2}$. Indeed, as shown in Figs. 10 and 11 of Ref. [9], a simplified version of our theory almost quantitatively fits with Monte Carlo data for the motion of individual segments. Furthermore, as will be illustrated in Sec. VIII and in more detail in Ref. [14], our theory quantitatively accounts for data for the coherent scattering function $S_{c}(q, t ; M, N)$. The agreement is equally good for the total chain $(M=N)$ where tube length fluctuations are very important, and for an interior piece $(M<N)$ where tube length fluctuations are irrelevant.

\section{Distribution function for $\mathcal{S}_{\mathbf{2}}(q, t ; j, k, N)$}

To evaluate $\mathcal{S}_{2}(q, t ; j, k, N)$ [Eq. (4.7)], we need the distribution function

$$
\begin{aligned}
\mathcal{P}_{j}^{(T)}\left(n_{j} ; t\right) & =\overline{\left[\Theta\left(j_{>}-j_{<}\right)-1\right] \delta_{n_{j}, n(j, t)}} \\
& =\sum_{n_{m}=0}^{\infty} \mathcal{P}_{\max , j}^{(T)}\left(n_{m}, n_{j} ; t\right)-\mathcal{P}_{1}\left(n_{j} ; j, t\right) .
\end{aligned}
$$

The first part can be determined by integrating $\mathcal{P}_{\max , j}^{(T)}[\mathrm{Eq}$. (4.26)] over $n_{m}$. Equations (4.26)-(4.28) thus yield

$$
\begin{gathered}
d n_{j} \mathcal{P}_{j}^{(T)}\left(n_{j} ; t\right)=\frac{d z_{j}}{\sqrt{2 \pi}} \sum_{\nu=-\infty}^{+\infty} \mathcal{P}_{j}^{(\nu)}\left(z_{j}, a\right), \\
\mathcal{P}_{j}^{(\nu)}\left(z_{j}, a\right)=\int_{0}^{\hat{N}} d y \mathcal{P}_{\max , j}^{(\nu)}\left(y, z_{j}, a\right)-\delta_{\nu, 0} \exp \left(-z_{j}^{2} / 2\right) .
\end{gathered}
$$


The $y$ integral can be evaluated analytically and we quote the result, which is useful for the numerical evaluation of $\mathcal{S}_{2}$, in Appendix B.

\section{E. Result for the tube conserving contribution to the coherent structure function $S_{c}^{(T)}(q, t ; M, N)$}

We consider the scattering from the $M+1$ central beads [Eq. (3.18)] and write

$$
\begin{aligned}
S_{c}^{(T)}(q, t ; M, N)= & \mathcal{S}_{1}(q, t ; M, N)+\mathcal{S}_{2}(q, t ; M, N) \\
& +\mathcal{S}_{3}(q, t ; M, N),
\end{aligned}
$$

where the $\mathcal{S}_{i}(q, t ; M, N), i=1,2,3$, are the contributions $\mathcal{S}_{i}(q, t ; j, k, N)$ [Eq. (4.5)], summed over $j$ and $k$. The superscript $(T)$ again recalls the constraint $(4.1), j_{>}-j_{<} \geqslant 0$.

Due to this constraint the relation

$$
S_{c}(q, t ; M, N)=S_{c}^{(T)}(q, t ; M, N)
$$

in general holds only for $t \ll T_{3}$. However, for large $q$ such that $q^{2} R_{g}^{2} \gg 1$, contributions in which the tube has been destroyed, contribute negligibly to $S_{c}(q, t ; M, N)$, so that Eq. (4.32) in this limit holds for all times.

Consider now the first contribution.

$$
\mathcal{S}_{1}(q, t ; M, N)=\int_{(N-M) / 2}^{(N+M) / 2} d j d k \mathcal{S}_{1}(q, t ; j, k, N) .
$$

Using Eqs. (4.10) and (4.11), we can carry out the integral over $k$ to find

$$
\begin{aligned}
\mathcal{S}_{1}(q, t ; M, N)= & \frac{1}{2 \bar{q}^{2}} \int_{(N-M) / 2}^{(N+M) / 2} d j\left[e^{2 \Delta_{1} Q+Q^{2}} \operatorname{erfc}\left(Q+\Delta_{1}\right)\right. \\
& -e^{-2 \Delta_{1} Q+Q^{2}} \operatorname{erfc}\left(Q-\Delta_{1}\right) \\
& -e^{2 \Delta_{2} Q+Q^{2}} \operatorname{erfc}\left(Q+\Delta_{2}\right) \\
& +e^{-2 \Delta_{2} Q+Q^{2}} \operatorname{erfc}\left(Q-\Delta_{2}\right) \\
& \left.+2 \operatorname{erfc} \Delta_{2}-2 \operatorname{erfc} \Delta_{1}\right]
\end{aligned}
$$

where

$$
\begin{gathered}
Q=\bar{q}^{2} \bar{l}_{s} \sqrt{\rho_{0} A_{1}(j, t)}, \\
\Delta_{1}=\frac{1}{2 \bar{l}_{s}}\left(\frac{N+M}{2}-j\right)\left[\rho_{0} A_{1}(j, t)\right]^{-1 / 2}, \\
\Delta_{2}=\frac{1}{2 \bar{l}_{s}}\left(\frac{N-M}{2}-j\right)\left[\rho_{0} A_{1}(j, t)\right]^{-1 / 2} .
\end{gathered}
$$

The remaining integration in general must be done numerically, due to the $j$ dependence of $A_{1}(j, t)$.

The integral over $k$ can be carried out also in $\mathcal{S}_{2}, \mathcal{S}_{3}$. We introduce the notation

$$
\hat{q}=\bar{q}^{2} c, \quad \hat{j}=j / c, \quad \hat{M}=M / c,
$$

$$
b=\frac{\bar{l}_{s}}{c} \overline{\left.n^{2}\left(\frac{N-M}{2}+j, t\right)\right]^{1 / 2}},
$$

where the parameter $c$ has been defined in Eq. (4.25). With due regard of the $\Theta$ functions, a straightforward calculation yields

$$
\mathcal{S}_{2}(q, t ; M, N)=\frac{c^{2}}{\hat{q}} \int_{0}^{\hat{M}} d \hat{j}\left(\mathcal{S}_{2}^{(1)}+\mathcal{S}_{2}^{(2)}\right)
$$

$$
\begin{aligned}
\mathcal{S}_{2}^{(1)}= & \frac{1}{b}\left(1-e^{-\hat{q} \hat{M}}\right) \int_{0}^{\infty} d z e^{-\hat{q} z} \frac{1}{\sqrt{2 \pi}} \sum_{\nu}\left[\mathcal{P}_{j}^{(\nu)}\left(-\frac{z+\hat{j}}{b}, a\right)\right. \\
+ & \left.\mathcal{P}_{j}^{(\nu)}\left(\frac{z-\hat{j}+\hat{N}}{b}, a\right)\right], \\
\mathcal{S}_{2}^{(2)}= & \frac{1}{b} \int_{0}^{\hat{M}} d z\left[2-e^{-\hat{q} z}-e^{-\hat{q}(\hat{M}-z)}\right] \frac{1}{\sqrt{2 \pi}} \\
& \times \sum_{\nu} \mathcal{P}_{j}^{(\nu)}\left(\frac{z-\hat{j}}{b}, a\right)
\end{aligned}
$$

$$
\mathcal{S}_{3}(q, t ; M, N)=\frac{2 c^{2}}{\hat{q}} \int_{0}^{\hat{M}} d \hat{j}\left(\mathcal{S}_{3}^{(1)}+\mathcal{S}_{3}^{(2)}+\mathcal{S}_{3}^{(3)}+\mathcal{S}_{3}^{(4)}\right),
$$

$$
\begin{aligned}
\mathcal{S}_{3}^{(1)}= & \frac{1}{b} \int_{0}^{(\hat{N}+\hat{M}) / 2} d y y \int_{0}^{1} d z\left[2 e^{-\hat{q} y(1-z)}-2+e^{-\hat{q} y z}\right. \\
& \left.-e^{-\hat{q} y(2-z)}\right] \frac{1}{\sqrt{2 \pi}} \sum_{\nu} \mathcal{P}_{\text {max }, j}^{(\nu)}\left(y+\frac{\hat{N}-\hat{M}}{2}, \frac{y}{b} z-\frac{\hat{j}}{b}, a\right),
\end{aligned}
$$

$$
\begin{aligned}
\mathcal{S}_{3}^{(2)}= & -\frac{1}{b} \int_{0}^{(\hat{N}+\hat{M}) / 2} d y \int_{0}^{\infty} d z e^{-\hat{q} z}\left(1-e^{-\hat{q} y}\right)^{2} \frac{1}{\sqrt{2 \pi}} \\
& \times \sum_{\nu} \mathcal{P}_{\max , j}^{(\nu)}\left(y+\frac{\hat{N}-\hat{M}}{2},-\frac{z+\hat{j}}{b}, a\right), \\
\mathcal{S}_{3}^{(3)}= & -\frac{1}{b} \int_{0}^{(\hat{N}-\hat{M}) / 2} d y y \int_{0}^{1} d z\left[2 e^{-\hat{q} y(1-z)}-2+e^{-\hat{q} y z}\right. \\
& -e^{-\hat{q} y(2-z)} \frac{1}{\sqrt{2 \pi}} \sum_{\nu} \mathcal{P}_{\max , j}^{(\nu)} \\
& \times\left(y+\frac{\hat{N}+\hat{M}}{2}, \frac{y}{b} z+\frac{\hat{M}-\hat{j}}{b}, a\right),
\end{aligned}
$$




$$
\begin{aligned}
\mathcal{S}_{3}^{(4)}= & \frac{1}{b} \int_{0}^{(\hat{N}-\hat{M}) / 2} d y \int_{0}^{\infty} d z e^{-\hat{q} z}\left(1-e^{-\hat{q} y}\right)^{2} \frac{1}{\sqrt{2 \pi}} \\
& \times \sum_{\nu} \mathcal{P}_{\max , j}^{(\nu)}\left(y+\frac{\hat{N}+\hat{M}}{2},-\frac{z}{b}+\frac{\hat{M}-\hat{j}}{b}, a\right) .
\end{aligned}
$$

The prefactor of 2 in Eq. (4.40) accounts for the last two contributions in Eq. (4.8). We note that these results depend on time via the parameters $a, c$, and $b$. From its definition (4.36), the parameter function $b=b(j, t)$ measures the motion of an arbitrary segment relative to the motion of the end segment. It is weakly dependent on $j$ and tends to 1 for $t$ $\gg T_{2}$.

In $\mathcal{S}_{2}$ and $\mathcal{S}_{3}$, one more integration could be done analytically, which, however, only blows up the number of terms without leading to any simplification. Due to the dependence on the segment index $j$ implicit in $a=a(j, t)$ and $b=b(j, t)$, an analytical evaluation of all integrals is possible only in the limit $t \gg T_{2}$ where $a \rightarrow 1$ and $b \rightarrow 1$. In general, we have to resort to numerical evaluation. In this context, we may note that the summations over $\nu$ for $t \lesssim T_{3}$ converge rapidly, so that in the range where $S_{c}^{(T)}(t \neq 0) / S_{c}(0)$ exceeds $10^{-3}$, we never need to go beyond $|\nu| \leqslant 4$.

\section{DYNAMICS WITHIN THE INITIAL TUBE}

In a time region where end effects are unimportant, the results of the preceding section can be simplified. In precise terms, the neglect of end effects amounts to considering a subchain of length $M$, in the center of an infinitely long chain. We here concentrate on this particular limit and compare our results to those derived for a Rouse chain in a coiled tube.

\section{A. Results of the reptation model}

In the limit $N \rightarrow \infty$, with $\tilde{j}=j-N / 2$ and $\tilde{k}=k-N / 2$ fixed, only the contribution $\mathcal{S}_{1}$ to $S^{(T)}(q, t ; j, k, N)$ [Eq. (4.5)] survives. Furthermore, $A_{1}(j, t)$ (3.5) simplifies to [see Ref. [8], Eq. (4.11)]

$$
\begin{aligned}
A_{1}(j, t) & =\frac{\hat{t}^{1 / 2}}{\pi} \int_{0}^{4 \hat{t}} \frac{d z}{\sqrt{z}} \sqrt{1-\frac{z}{4 \hat{t}}} e^{-z} \\
& \hat{t} \gg 1 \\
& \rightarrow \sqrt{\frac{\hat{t}}{\pi}}
\end{aligned}
$$

independent of $j$. (Recall the definition $\hat{t}=p t$.) $\mathcal{S}_{1}(q, t ; j, k, N)[$ Eq. (4.10)] takes the form

$$
\begin{aligned}
& \mathcal{S}_{1}(q, t ; \tilde{j}, \widetilde{k})=\frac{1}{2} e^{\hat{Q}^{2}}\left[e^{2 \hat{\Delta} \hat{Q}} \operatorname{erfc}(\hat{Q}+\hat{\Delta})\right. \\
& \left.+e^{-2 \hat{\Delta} \hat{Q}} \operatorname{erfc}(\hat{Q}-\hat{\Delta})\right],
\end{aligned}
$$

where now

$$
\begin{aligned}
& \hat{Q}=\bar{q}^{2} \sqrt{\bar{l}_{s}^{2} \rho_{0}}\left(\frac{\hat{t}}{\pi}\right)^{1 / 4}, \\
& \hat{\Delta}=\frac{\tilde{k}-\tilde{j}}{2 \sqrt{\bar{l}_{s}^{2} \rho_{0}}}\left(\frac{\hat{t}}{\pi}\right)^{-1 / 4} .
\end{aligned}
$$

Integrating over $\tilde{j}$ and $\widetilde{k}$, we find for the normalized dynamic structure function

$$
\begin{aligned}
& \bar{S}_{c}(q, t ; M, \infty)=\frac{S_{c}(q, t ; M, \infty)}{M^{2} D\left(\bar{q}^{2} M\right)} \\
& =1-\frac{1}{\bar{q}^{2} M D\left(\bar{q}^{2} M\right)}\left\{2 \operatorname{erfc}\left[\left(\frac{\hat{T}_{4}}{\hat{t}}\right)^{1 / 4}\right]\right. \\
& \left.+\frac{2}{\sqrt{\pi}}\left(\frac{\hat{t}}{\hat{T}_{4}}\right)^{1 / 2}\left(1-e^{-\left(\hat{T}_{4} / \hat{t}\right)^{1 / 2}}\right)\right\} \\
& +\frac{1}{\left(\bar{q}^{2} M\right)^{2} D\left(\bar{q}^{2} M\right)}\left\{2-2 e^{-\bar{q}^{2} M}\right. \\
& +e^{\hat{Q}^{2}}\left\{e^{\bar{q}^{2} M} \operatorname{erfc}\left[\hat{Q}+\left(\frac{\hat{T}_{4}}{\hat{t}}\right)^{1 / 4}\right]\right. \\
& \left.\left.+e^{-\bar{q}^{2} M} \operatorname{erfc}\left[\hat{Q}-\left(\frac{\hat{T}_{4}}{\hat{t}}\right)^{1 / 4}\right]-2 \operatorname{erfc}(\hat{Q})\right\}\right\} \text {. }
\end{aligned}
$$

We recall that $D(x)=\left(2 / x^{2}\right)\left(e^{-x}-1+x\right)$ is the Debye function, and note that $\bar{q}^{2} M$ can also be written as

$$
\bar{q}^{2} M=q^{2} R_{g}^{2}(M)
$$

where $R_{g}(M)$ is the radius of gyration of the subchain. In Eq. (5.4), we introduced a new time scale

$$
\hat{T}_{4}=\frac{\pi}{16}\left(\bar{l}_{s}^{2} \rho_{0}\right)^{-2} M^{4}
$$

which is of the order of the time the subchain needs to leave its original part of the tube. This interpretation is obvious from the results on segment motion quoted at the end of Sec. II, $\left\langle\left[r_{j}\left(\hat{T}_{4}\right)-r_{j}(0)\right]^{2}\right\rangle \sim \hat{T}_{4}^{1 / 4} \sim M$ for $\hat{T}_{4} \ll \hat{T}_{2}$. Also the variable $\hat{Q}$ can be expressed in terms of a time scale,

$$
\begin{gathered}
\hat{Q}=\left(\frac{\hat{t}}{\hat{T}_{q}}\right)^{1 / 4}, \\
\hat{T}_{q}=\pi\left(\bar{l}_{s}^{2} \rho_{0}\right)^{-2}\left(\bar{q}^{2}\right)^{-4}=\frac{16 \hat{T}_{4}}{\left[q^{2} R_{g}^{2}(M)\right]^{4}} .
\end{gathered}
$$

It needs the time $\hat{T}_{q}$ before the distance diffused by the subchain, can be resolved by scattering of wave vector $q$. For comparison with previous work, we concentrate on 
$q^{2} R_{g}^{2}(M) \gg 1$, so that $\hat{T}_{q} \ll \hat{T}_{4}$ and $D(x) \approx 2 / x$. We then find the following limiting behavior in the various time regimes:

$$
\begin{aligned}
& \frac{S_{c}(q, t ; M, \infty)}{M^{2}}-D\left[q^{2} R_{g}^{2}(M)\right] \\
& \approx\left\{\begin{array}{l}
-2 \frac{1-e^{-q^{2} R_{g}^{2}(M)}}{\left[q^{2} R_{g}^{2}(M)\right]^{2}}\left(\frac{\hat{t}}{\hat{T}_{q}}\right)^{1 / 2} \text { for } \hat{t} \ll \hat{T}_{q} \\
-\frac{2}{\sqrt{\pi} q^{2} R_{g}^{2}(M)}\left(\frac{\hat{t}}{\hat{T}_{4}}\right)^{1 / 4} \text { for } \hat{T}_{q} \ll \hat{t} \ll \hat{T}_{4},
\end{array}\right. \\
& \frac{S_{c}(q, t ; M, \infty)}{M^{2}} \approx \frac{6}{\sqrt{\pi} q^{2} R_{g}^{2}(M)}\left(\frac{\hat{T}_{4}}{\hat{t}}\right)^{1 / 4} \text { for } \hat{T}_{4} \ll \hat{t} .
\end{aligned}
$$

Note that our results depend only on macroscopic parameters $q^{2} R_{g}^{2}(M)$ and $\hat{t} / \hat{T}_{4}$, which absorb any reference to the microscopic structure.

\section{B. Comparison with de Gennes' results for Rouse motion in a tube}

In Sec. II B, we recalled de Gennes' results [12], derived for one-dimensional Rouse-type motion in a coiled tube. In the derivation, the relation $q^{2} R_{g}^{2} \gg 1 \gg q^{2} l^{2}$ was assumed, with $l$ being the average segment size of a Gaussian chain. Of interest here is the "local" term $\bar{S}^{(l)}(q, t)$ [Eq. (2.12)]. A glance at the derivation shows that it implicitly exploits the limit considered here: a subchain (of length $M$ ) in an infinitely long chain. Combining Eqs. (2.10) to Eq. (2.14) we thus find

$$
\begin{gathered}
\frac{S_{d G}(q, t ; M, \infty)}{M^{2} D\left(\bar{q}^{2} M\right)}=1-N_{e} \bar{q}^{2}\left[1-e^{\left.t / T_{q} \operatorname{erfc}\left(\sqrt{t / T_{q}}\right)\right]}\right. \\
T_{q}=\frac{\text { const }}{\left(\bar{q}^{2}\right)^{2}} .
\end{gathered}
$$

Clearly this expression differs strongly from our result (5.4). It leads to very different asymptotics,

$$
\begin{aligned}
& \frac{S_{d G}(q, t ; M, \infty)}{M^{2}}-D\left[q^{2} R_{g}^{2}(M)\right] \\
& =\frac{2 N_{e}}{M}\left\{\begin{array}{l}
-\frac{2}{\sqrt{\pi}}\left(\frac{t}{T_{q}}\right)^{1 / 2} \text { for } t \ll T_{q} \\
-1+\left(\frac{T_{q}}{\pi t}\right)^{1 / 2} \text { for } t \gg T_{q} .
\end{array}\right.
\end{aligned}
$$

Furthermore the scaling with $q^{2}, M$, and $t$ is quite different.

\section{Closer inspection of Rouse motion in a tube}

The derivation of Eq. (5.11) in Ref. [12] involves some approximations, which greatly simplify the analysis but are not really necessary. For a general test of the validity of the model, we therefore have repeated the analysis without these simplifications. The analysis is sketched in Appendix C. For further discussion, we here quote the result for the scattering from a given pair of beads [Eqs. (C16)-(C18)],

$$
\begin{aligned}
& S_{R T}(q, t ; \tilde{j}, \widetilde{k})=\frac{1}{2} e^{\widetilde{Q}^{2}}\left\{e^{2 \hat{\Delta} \tilde{Q}} \operatorname{erfc}(\widetilde{Q}+\widetilde{\Delta})\right. \\
& \left.+e^{-2 \hat{\Delta} \tilde{Q}} \operatorname{erfc}(\widetilde{Q}-\widetilde{\Delta})\right\} \\
& \widetilde{Q}=q^{2} l^{2} \sqrt{\frac{N_{e}}{6}}\left[|\tilde{j}-\widetilde{k}|+\sqrt{2 \frac{\gamma_{0}}{l^{2}}} \operatorname{tg}\left(\frac{|\tilde{j}-\tilde{k}|}{\sqrt{2 \frac{\gamma_{0}}{l^{2}} t}}\right)\right]^{1 / 2}, \\
& \tilde{\Delta}=\sqrt{\frac{6}{N_{e}}} \frac{|\tilde{j}-\tilde{k}|}{2}\left[|\tilde{j}-\tilde{k}|+\sqrt{2 \frac{\gamma_{0}}{l^{2}}} \operatorname{tg}\left(\frac{|\tilde{j}-\tilde{k}|}{\sqrt{2 \frac{\gamma_{0}}{l^{2}} t}}\right)\right]^{-1 / 2}, \\
& g(z)=\frac{1}{\sqrt{\pi}} e^{-z^{2}}-z \operatorname{erfc} z
\end{aligned}
$$

Here $\gamma_{0}$ is the segment mobility of the one-dimensional Rouse model.

Clearly the structure of $S_{R T}$ [Eq. (5.14)] is identical to that of our result $\mathcal{S}_{1}$ [Eq. (5.2)]. The difference is in the quantities $\widetilde{Q}, \widetilde{\Delta}$ [Eq. (5.15)], compared to $\hat{Q}, \hat{\Delta}$ [Eq. (5.3)]. We note, however, that the relation

$$
2 \widetilde{Q} \widetilde{\Delta}=q^{2} l^{2}|\widetilde{j}-\widetilde{k}|=q^{2} \frac{l_{0}^{2}}{6}|\widetilde{j}-\widetilde{k}|=2 \hat{Q} \hat{\Delta}
$$

holds. Recall that the mean segment size $l$ of a Gaussian chain, which is asymptotically equivalent to a chain with fixed segment length $l_{0}$, obeys $l^{2}=l_{0}^{2} / 6$ (in three dimensions).

To analyze the difference among the two models, we first consider the static limit, $t=0 . \mathcal{S}_{1}$ reduces to (recall the definition $\bar{q}^{2}=q^{2} l_{0}^{2} / 6 \equiv q^{2} l^{2}$ )

$$
\mathcal{S}_{1}(q, 0 ; \tilde{j}, \tilde{k})=e^{-q^{2} l^{2}|\tilde{j}-\tilde{k}|}
$$




$$
\begin{aligned}
S_{R T}(q, 0 ; \tilde{j}, \tilde{k})= & \exp \left[-q^{2} l^{2}|\tilde{j}-\widetilde{k}|\left(1-q^{2} l^{2} \frac{N_{e}}{6}\right)\right] \\
& \times\left\{1-\frac{1}{2} \operatorname{erfc}\left[\sqrt{\frac{6}{N_{e}}|\tilde{j}-\tilde{k}|}\left(\frac{1}{2}-q^{2} l^{2} \frac{N_{e}}{6}\right)\right]\right\} \\
& +\frac{1}{2} \exp \left[q^{2} l^{2}|\tilde{j}-\widetilde{k}|\left(1+q^{2} l^{2} \frac{N_{e}}{6}\right)\right] \\
& \times \operatorname{erfc}\left[\sqrt{\frac{6}{N_{e}}|\tilde{j}-\widetilde{k}|}\left(\frac{1}{2}+q^{2} l^{2} \frac{N_{e}}{6}\right)\right] .
\end{aligned}
$$

Even if we ignore the terms $q^{2} l^{2} N_{e} / 6 \ll 1$, taking them to be irrelevant microstructure effects, this result does not reduce to the exact form (5.17). To recover this form, we rather consistently have to take the limit $q^{2} l^{2} \rightarrow 0$ with $q^{2} l^{2}(\tilde{j}-\widetilde{k})$ fixed, i.e., $|\tilde{j}-\widetilde{k}| \equiv|j-k| \rightarrow \infty$. This just demonstrates that a one-dimensional Gaussian chain, folded into the threedimensional random walk configuration of the tube, does not yield the exact distribution of a three-dimensional Gaussian chain. In other words, the model of a Rouse chain in a tube violates the equilibrium initial conditions by microstructure terms on scale $|\tilde{j}-\widetilde{k}| \sim N_{e}$.

For the dynamics, this discussion implies that the model gives a wrong estimate for the number of wiggles in the initial configuration. To eliminate this effect of unphysical initial conditions, we have to take the same limit $q^{2} l^{2} \rightarrow 0$ with $q^{2} l^{2}(\tilde{j}-\widetilde{k})$ fixed, also in the full time dependent expression (5.14). To facilitate the discussion, we rewrite Eqs. (5.15) in a form that exhibits the fixed combination of variables $q^{2} l^{2}|\widetilde{j}-\widetilde{k}|$ :

$$
\begin{aligned}
\widetilde{Q}= & q l \sqrt{\frac{N_{e}}{6}}\left[q^{2} l^{2}|\tilde{j}-\tilde{k}|\right. \\
& \left.+q^{2} l^{2} \sqrt{2 \frac{\gamma_{0}}{l^{2}}} \operatorname{tg}\left(\frac{q^{2} l^{2}|\tilde{j}-\tilde{k}|}{q^{2} l^{2} \sqrt{2 \frac{\gamma_{0}}{l^{2}} t}}\right)\right]^{1 / 2}, \\
\widetilde{\Delta}= & \frac{1}{2 q l} \sqrt{\frac{6}{N_{e}}} q^{2} l^{2}|\tilde{j}-\widetilde{k}|\left[q^{2} l^{2}|\tilde{j}-\tilde{k}|\right. \\
& \left.+q^{2} l^{2} \sqrt{2 \frac{\gamma_{0}}{l^{2}}} \operatorname{tg}\left(\frac{q^{2} l^{2}|\tilde{j}-\tilde{k}|}{q^{2} l^{2} \sqrt{2 \frac{\gamma_{0}}{l^{2}}} t}\right)\right]^{-1 / 2} .
\end{aligned}
$$

The function $g(z)$ obeys the relations

$$
\begin{gathered}
g(0)=\frac{1}{\sqrt{\pi}}, \\
g(z) \sim e^{-z^{2}}, \quad z \rightarrow \infty .
\end{gathered}
$$

Now the limiting result for $S_{R T}$ sensitively depends on the way we scale the time. We first consider times such that $q^{2} \sqrt{t}$ stays finite upon taking the limit $q^{2} l^{2} \rightarrow 0$,

$$
q^{2} l^{2} \sqrt{2 \frac{\gamma_{0}}{l^{2}}} t \sim q^{\alpha}, \quad \alpha \geqslant 0 .
$$

We then find

$$
\widetilde{Q} \rightarrow 0, \quad \widetilde{\Delta} \rightarrow \infty
$$

and recover the static limit (5.17). Indeed, for such times the scattering cannot resolve the internal motion,

$$
q^{2}\left\langle\overline{\left[\mathbf{r}_{j}(t)-\mathbf{r}_{j}(0)\right]}\right\rangle \sim q^{2} t^{1 / 4} \sim q^{1+\alpha / 2} \rightarrow 0 .
$$

Effects of internal dynamics can be seen only for times such that $q^{2} \sqrt{t}$ diverge,

$$
q^{2} l^{2} \sqrt{2 \frac{\gamma_{0}}{l^{2}} t} \sim q^{-\alpha}, \quad \alpha>0 .
$$

Then the contribution proportional to $g$ dominates the square brackets in Eqs. (5.19), and furthermore the argument of $g$ tends to zero. Thus

$$
\begin{gathered}
\widetilde{Q} \rightarrow q^{2} l^{2} \sqrt{\frac{N_{e}}{6}}\left(\frac{2}{\pi} \frac{\gamma_{0}}{l^{2}} t\right)^{1 / 4}, \\
\widetilde{\Delta} \rightarrow \frac{1}{2} \sqrt{\frac{6}{N_{e}}}|\widetilde{j}-\widetilde{k}|\left(\frac{2}{\pi} \frac{\gamma_{0}}{l^{2}} t\right)^{-1 / 4},
\end{gathered}
$$

which is the same functional dependence on $t$ and $|\widetilde{j}-\widetilde{k}|$ as that of $\hat{Q}$ or $\hat{\Delta}$ [Eq. (5.3)]. The limiting expression for $S_{R T}(q, t ; \tilde{j}, \widetilde{k})$ becomes identical to our result $\mathcal{S}_{1}(q, t ; \tilde{j}, \widetilde{k})$ if we identify

$$
\begin{gathered}
q^{2} l^{2}=\bar{q}^{2}=q^{2} \frac{l_{0}^{2}}{6}, \\
\frac{N_{e}^{2}}{18} \frac{\gamma_{0}}{l^{2}} t=\left(\bar{l}_{s}^{2} \rho_{0}\right)^{2} \hat{t} .
\end{gathered}
$$

In summary, we have found that the model of a Rouse chain in a tube is equivalent to the reptation model only in the limit $q^{2} l^{2} \rightarrow 0$ with $q^{2} l^{2}(j-k)$ fixed. Outside this limit, it exhibits an unphysical relaxation of nonequilibrium initial conditions. 


\section{Relation among the microscopic parameters of the different models}

As a byproduct of our analysis, we with Eq. (5.22) have found a relation among the microscopic parameters of our model and those used in more standard Rouse type modeling of chain dynamics. Analyzing in Sec. VII the relation of our model with the primitive chain model, we will find as an additional result,

$$
\frac{N_{e}}{6} \frac{\gamma_{0}}{l^{2}} t=\bar{l}_{s}^{2} \rho_{0} \hat{t} .
$$

Combining Eqs. (5.22) and (5.23), we find

$$
\begin{gathered}
N_{e}=3 \bar{l}_{s}^{2} \rho_{0}, \\
\frac{\gamma_{0}}{2 l^{2}} t=\hat{t} .
\end{gathered}
$$

We now can give a quantitative definition of the equilibration time $T_{2}$, which we identify with the Rouse time of a free chain of $N$ segments,

$$
T_{2}=\frac{2}{\pi^{2}}(N+1)^{2} \frac{l^{2}}{\gamma_{0}} .
$$

With Eq. (5.25), we find

$$
\hat{T}_{2}=p T_{2}=\frac{(N+1)^{2}}{\pi^{2}} .
$$

\section{E. Implications for the coherent structure function}

The artifact of the model of a Rouse chain in a tube concerns only small parts of the chain of the order of the tube diameter and thus might be thought to be negligible. In the static structure function, the error sums up to a term of order $N$, small compared to $S_{c}(q, 0 ; N)=N^{2} D\left(\bar{q}^{2} N\right)$. Since, however, for $t \leqq T_{2}$ the time dependence of $S_{c}$ is weak, even such a small effect is relevant. Indeed, it can greatly change the picture. To illustrate this, we in Fig. 3 compare our result (5.4) for the normalized coherent structure function $S_{c}(q, t ; M, \infty)$ to the result found by integrating $S_{R T}(q, t ; \tilde{j}, \widetilde{k})$ [Eq. (5.14)] over $-M / 2 \leqslant \tilde{j} \leqslant M / 2$ and $-M / 2 \leqslant \tilde{k} \leqslant M / 2$. To relate the models, we used relations (5.21), (5.24), and (5.25). To include also de Gennes' approximate form (5.11), we used the large value $q^{2} R_{g}^{2}(M)=50$. We note that de Gennes enforced the correct $t=0$ behavior by artificially subtracting his result for $S_{R T}$. We furthermore note that repeating his calculation in our notation, we found $t / T_{q}=\bar{q}^{4} \hat{t}$. The remaining parameter $\bar{l}_{s}^{2} \rho_{0}=1.23$ has been taken from our previous work [9].

Figure 3 shows that the effect of the artificial initial conditions can be quite large and dies out only slowly. It extends up to the Rouse time of the subchain considered. This result is generic. For longer chains, the amplitude of the effect decreases for the normalized structure function, as expected

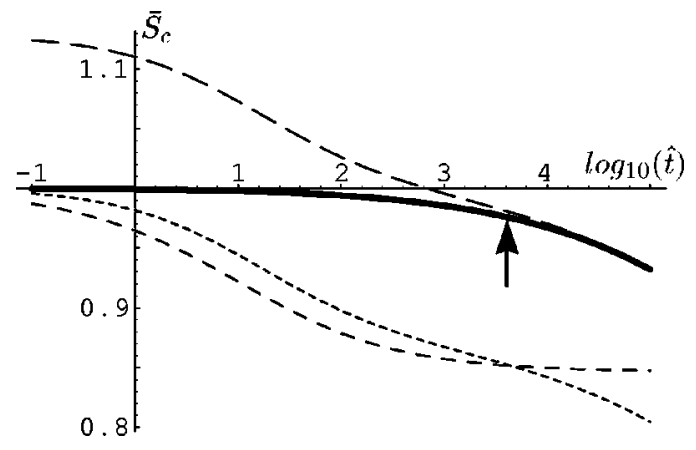

FIG. 3. Coherent normalized structure function $\bar{S}_{c}(q, t ; M, \infty)$ of the central piece of an infinitely long chain for $q^{2} R_{g}^{2}(M)=50, M$ $=200$. Thick line, reptation result [Eq. (5.4)]; long dashes and short dashes, Rouse chain in a coiled tube. For the latter curve, a constant has been subtracted such that $\bar{S}_{c}(q, 0 ; M, \infty)=1$. Medium size dashes, de Gennes' approximation [Eq. (5.11)]. The arrow points to $\hat{T}_{2}(M=200)$.

for a microstructure effect, but the range stays of order $T_{2}(M)$. This is obvious since a nonequilibrium initial condition generically will relax only on time scale $T_{2}$. As a side issue, we note that de Gennes' approximation (5.11) agrees quite well with the shifted form of $S_{R T}$.

To close this section, a general remark on microstructure corrections for the dynamics may be appropriate. Our result shows no such corrections, which would give rise to an additional $1 / M$ dependence in Eq. (5.4), which is not in the form of the scaling variables $\bar{q}^{2} M$ and $\hat{t} / \hat{T}_{4}$. Thus our model succeeded in singling out the universal aspects of reptation dynamics. This, however, does not imply that (nonuniversal) terms yielding some additional $1 / M$ dependence could not show up for a microscopically realistic model, which takes the details of the microscopic motion into account. But we want to stress that any model first of all has to yield the correct static structure function. Otherwise some unphysical relaxation will influence the dynamics. Such results can safely be trusted only in a range where $S_{c}(q, 0 ; N)$ $-S_{c}(q, t ; N)$ exceeds the error in $S_{c}(q, 0 ; N)$.

We finally note that here we have been concerned exclusively with the reptation aspect of the dynamics, modeled as one-dimensional Rouse motion in a tube. This is to be clearly distinguished from three-dimensional Rouse motion among fixed entanglement points, as treated by Des Cloizeaux [15], for instance. The latter model is concerned with motion in melts for "microscopic" times, $t \lesssim T_{0}$.

\section{ANALYSIS OF COMPLETE TUBE DESTRUCTION}

In this section, we derive an integral equation extending $S_{c}(q, t ; M, N)$ to arbitrarily large times (Sec. VI A). Basically it is an equation for $S(q, t ; j, k, N)$, which incorporates $S^{(T)}(q, t ; j, 0, N)$ as inhomogeneity. To calculate $S_{c}$, we need to sum this inhomogeneity over $j$. We construct this function in Sec. VIB, following the approach of Sec. IV. The kernel of the integral equation involves some distribution function which is calculated in Sec. VIC with the help of the mean hopping rate approximation. Quantities like the probability 


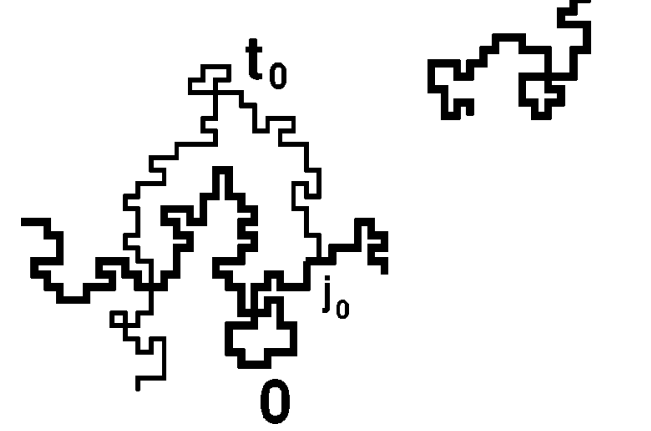

FIG. 4. Initial (0) and final $(t)$ configuration of the chain (thick lines), together with the configuration at time $t_{0}$ (thin line). At time $t_{0}$, the chain leaves the last piece of the initial tube, with one chain end at the position of bead $j_{0}$ in the initial tube.

density of tube destruction at time $t$, which can be derived from this distribution function, are discussed in Sec. VID. The numerical evaluation of our results for $S_{c}(q, t ; M, N)$ is deferred to Sec. VIII, after we have shown that our theory in the appropriate limit yields the results of the primitive chain model.

\section{A. Derivation of an integral equation for the structure function}

Up to now, we only considered stochastic processes for which some part of the initial tube still exists at time $t$. To get rid of this constraint, we have to deal with situations as shown in Fig. 4: at time $t_{0}, 0<t_{0}<t$, the chain leaves the original tube, which means that the remainder of the original tube is the single point $\mathbf{r}_{j_{0}}(0)$. This point is occupied by a chain end. The rest of the chain has found a completely new configuration.

Let $\mathcal{P}^{*}\left(j_{0}, t_{0} \mid 0\right)$ or $\mathcal{P}^{*}\left(j_{0}, t_{0} \mid N\right)$ be the probability that the tube is finally destroyed at time $t_{0}$, the last point $\mathbf{r}_{j_{0}}(0)$ being occupied by chain end 0 or $N$, respectively. We assume that $\mathcal{P}^{*}$ does not depend on the initial configuration, which should be satisfied except for rare extreme cases.

We then can write the full time dependent scattering (3.17) from a pair of beads $j, k$ as

$$
\begin{aligned}
S(q, t ; j, k, N)= & S^{(T)}(q, t ; j, k, N) \\
& +\sum_{t_{0}=1}^{t-1} \sum_{j_{0}=0}^{N}\left[\mathcal{P}^{*}\left(j_{0}, t_{0} \mid 0\right) S\left(q, t, j, k \mid j_{0}, t_{0}, 0\right)\right. \\
& \left.+\mathcal{P}^{*}\left(j_{0}, t_{0} \mid N\right) S\left(q, t, j, k \mid j_{0}, t_{0}, N\right)\right]
\end{aligned}
$$

where $S\left(q, t, j, k \mid j_{0}, t_{0}, m\right)$ with $m=0, N$ denotes the scattering with tube destruction specified by $j_{0}, t_{0}$, and $m$. We now factorize $S\left(q, t, j, k \mid j_{0}, t_{0}, m\right)$ according to

$$
\begin{aligned}
& S\left(q, t, j, k \mid j_{0}, t_{0}, m\right) \\
& =\left.\left\langle\overline{e^{i \mathbf{q} \cdot\left[\mathbf{r}_{j}(t)-\mathbf{r}_{k}(0)\right]}}\right\rangle\right|_{j_{0}, t_{0}, m} \\
& =\left.\left\langle\overline{e^{i \mathbf{q} \cdot\left[\mathbf{r}_{j}(t)-\mathbf{r}_{m}\left(t_{0}\right)\right]} e^{i \mathbf{q} \cdot\left[\mathbf{r}_{j_{0}}(0)-\mathbf{r}_{k}(0)\right]}}\right\rangle\right|_{j_{0}, t_{0}, m} \\
& \approx\left\langle\overline{e^{i \mathbf{q} \cdot\left[\mathbf{r}_{j}(t)-\mathbf{r}_{m}\left(t_{0}\right)\right]}}\right\rangle\left\langle e^{i \mathbf{q} \cdot\left[\mathbf{r}_{j_{0}}(0)-\mathbf{r}_{k}(0)\right]}\right\rangle, \quad m=0, N,
\end{aligned}
$$

where the second factor in the last line is a purely static average. We have exploited $r_{m}\left(t_{0}\right)=r_{j_{0}}(0)$. This factorization should be well justified, since the chain at time $t_{0}$ has attained a completely new internal configuration. Now the first factor in the last line of Eq. (6.2) equals $S(q, t$ $\left.-t_{0} ; j, m, N\right)$, whereas the second factor is the static structure function $\exp \left[-\bar{q}^{2}\left|j_{0}-k\right|\right]$. Combining Eqs. (6.1) and (6.2) we thus find

$$
\begin{aligned}
S(q, t ; j, k, N)= & S^{(T)}(q, t ; j, k, N) \\
& +\sum_{t_{0}=1}^{t-1} \sum_{j_{0}}\left[\mathcal{P}^{*}\left(j_{0}, t_{0} \mid 0\right) e^{-\bar{q}^{2}\left|j_{0}-k\right|}\right. \\
& \times S\left(q, t-t_{0} ; j, 0, N\right)+\mathcal{P}^{*}\left(j_{0}, t_{0} \mid N\right) e^{-\bar{q}^{2}\left|j_{0}-k\right|} \\
& \left.\times S\left(q, t-t_{0} ; j, N, N\right)\right] .
\end{aligned}
$$

Reflection symmetry along the chain implies

$$
\begin{gathered}
\mathcal{P}^{*}\left(j_{0}, t_{0} \mid N\right)=\mathcal{P}^{*}\left(N-j_{0}, t_{0} \mid 0\right), \\
S(q, t ; j, N, N)=S(q, t ; N-j, 0, N),
\end{gathered}
$$

so that our result takes the form

$$
\begin{aligned}
S(q, t ; j, k, N)= & S^{(T)}(q, t ; j, k, N)+\sum_{t_{0}=1}^{t-1} \sum_{j_{0}} \mathcal{P}^{*}\left(j_{0}, t_{0} \mid 0\right) \\
& \times\left[e^{-\bar{q}^{2}\left|j_{0}-k\right|} S\left(q, t-t_{0} ; j, 0, N\right)\right. \\
& \left.+e^{-\bar{q}^{2}\left|N-j_{0}-k\right|} S\left(q, t-t_{0} ; N-j, 0, N\right)\right] .
\end{aligned}
$$

We now sum $j$ and $k$ over the central piece of the chain to find

$$
\begin{aligned}
S_{c}(q, t & ; M, N) \\
= & S_{c}^{(T)}(q, t ; M, N) \\
& +2 \sum_{t_{0}=1}^{t-1}\left\{\sum_{j_{0}=0}^{N} \sum_{k=(N-M) / 2}^{(N+M) / 2} \mathcal{P}^{*}\left(j_{0}, t_{0} \mid 0\right) e^{-\bar{q}^{2}\left|j_{0}-k\right|}\right\} \\
& \times S_{E}\left(q, t-t_{0} ; M, N\right),
\end{aligned}
$$

where

$$
S_{E}(q, t ; M, N)=\sum_{j=(N-M) / 2}^{(N+M) / 2} S(q, t ; j, 0, n) .
$$


For $S_{E}$, Eq. (6.3) yields

$$
\begin{aligned}
S_{E}(q, t ; M, N)= & S_{E}^{(T)}(q, t ; M, N)+\sum_{t_{0}=1}^{t-1}\left\{\sum_{j_{0}=0}^{N} \mathcal{P}^{*}\left(j_{0}, t_{0} \mid 0\right)\right. \\
& \left.\times\left(e^{-\bar{q}^{2} j_{0}}+e^{-\bar{q}^{2}\left(N-j_{0}\right)}\right)\right\} S_{E}\left(q, t-t_{0} ; M, N\right) .
\end{aligned}
$$

With time (and segment index) taken continuous, this is the basic integral equation of our approach. We note that it is of Volterra type and therefore has a unique solution.

\section{B. Expression for $S_{E}^{(T)}(q, t ; M, N)$}

The tube conserving contribution to $S_{E}(q, t ; M, N)$ is easily found from the results of Sec. IV. Following Eq. (4.31), we write

$$
\begin{aligned}
& S_{E}^{(T)}(q, t ; M, N)=\mathcal{S}_{E, 1}(q, t ; M, N)+\mathcal{S}_{E, 2}(q, t ; M, N) \\
& +\mathcal{S}_{E, 3}(q, t ; M, N), \\
& \mathcal{S}_{E, 1}(q, t ; M, N)=\int_{(N-M) / 2}^{(N+M) / 2} d j \mathcal{S}_{1}(q, t ; j, 0, N), \\
& \mathcal{S}_{E, 2}(q, t ; M, N)=c \int_{(\hat{N}-\hat{M}) / 2}^{(\hat{N}+\hat{M}) / 2} d \hat{j} \int_{0}^{\infty} d z \frac{e^{-\hat{q} z}}{b} \frac{1}{\sqrt{2 \pi}} \\
& \times \sum_{\nu}\left[\mathcal{P}_{j}^{(\nu)}\left(\frac{z-\hat{j}}{b}, a\right)+\mathcal{P}_{j}^{(\nu)}\left(\frac{-z-\hat{j}}{b}, a\right)\right], \\
& \mathcal{S}_{E, 3}(q, t ; M, N)=c \int_{(\hat{N}-\hat{M}) / 2}^{(\hat{N}+\hat{M}) / 2} d \hat{j} \frac{1}{b} \int_{0}^{\hat{N}} d y\left\{\int _ { 0 } ^ { \infty } d z \left[e^{-\hat{q}(2 y+z)}\right.\right. \\
& \left.-e^{-\hat{q} z}\right] \frac{1}{\sqrt{2 \pi}} \sum_{\nu} \mathcal{P}_{\max , j}^{(\nu)}\left(y,-\frac{z+\hat{j}}{b}, a\right) \\
& +\int_{0}^{1} z y\left[e^{-\hat{q} y(2-z)}-e^{-\hat{q} y z}\right] \frac{1}{\sqrt{2 \pi}} \\
& \left.\times \sum_{\nu} \mathcal{P}_{\max , j}^{(\nu)}\left(y, \frac{y z-\hat{j}}{b}, a\right)\right\} .
\end{aligned}
$$

The notation is the same as in Sec. IV (see, in particular, Eqs. (4.27), (4.35), and (4.36)).

\section{Expression for $\mathcal{P}^{*}\left(j_{0}, t_{0} \mid \mathbf{0}\right)$}

To construct an expression for $\mathcal{P}^{*}\left(j_{0}, t_{0} \mid 0\right)$, we again use random walk theory, closely following the derivation of $\mathcal{P}_{\max , 0}^{(T)}$ in Sec. IV C. The calculation is sketched in Appendix A. It yields the result

$$
\begin{aligned}
d t_{0} \mathcal{P}^{*}\left(j_{0}, t_{0} \mid 0\right)= & \frac{d t_{0}}{t_{0}-1}\left[\pi p^{\prime}\left(t_{0}-1\right)\right]^{-1 / 2} \\
& \times \sum_{\nu=-\infty}^{+\infty} \nu\left[1-\frac{2\left(\nu N+j_{0} / 2\right)^{2}}{\bar{l}_{s}^{2} p^{\prime}\left(t_{0}-1\right)}\right] \\
& \times \exp \left[-\frac{\left(\nu N+j_{0} / 2\right)^{2}}{\bar{l}_{s}^{2} p^{\prime}\left(t_{0}-1\right)}\right] .
\end{aligned}
$$

We again express $t_{0}-1 \approx t_{0}$ in terms of the maximal excursion $\bar{n}_{m}$ [Eq. (4.21)] and identify $\bar{n}_{m}$ with $\overline{n_{\max }\left(0, t_{0}\right)}$. This yields the replacement

$$
\bar{l}_{s} \sqrt{p^{\prime}\left(t_{0}-1\right)} \rightarrow \frac{\sqrt{\pi}}{2} \bar{l}_{s} \overline{n_{\max }\left(0, t_{0}\right)}=\frac{c}{\sqrt{2}},
$$

resulting in

$$
\begin{gathered}
\frac{N}{\bar{l}_{s} \sqrt{p^{\prime}\left(t_{0}-1\right)}} \rightarrow \sqrt{2} \hat{N}=\sqrt{2} \frac{N}{c}, \\
\frac{j_{0}}{\bar{l}_{s} \sqrt{p^{\prime}\left(t_{0}-1\right)}} \rightarrow \sqrt{2} \hat{j}_{0}=\sqrt{2} \frac{j_{0}}{c}, \\
\frac{d t_{0}}{t_{0}-1} \rightarrow 2 \frac{d c}{c}, \quad \frac{d j_{0}}{\bar{l}_{s} \sqrt{\pi p^{\prime}\left(t_{0}-1\right)}} \rightarrow \sqrt{\frac{2}{\pi}} d \hat{j}_{0} .
\end{gathered}
$$

With these replacements, we find

$$
\begin{aligned}
d j_{0} d t_{0} \mathcal{P}^{*}\left(j_{0}, t_{0} \mid 0\right) \rightarrow & 2 \sqrt{\frac{2}{\pi}} \frac{d c}{c} d \hat{j}_{0} \sum_{\nu=-\infty}^{+\infty} \nu[1-4(\nu \hat{N} \\
& \left.\left.+\hat{j}_{0} / 2\right)^{2}\right] \exp \left[-2\left(\nu \hat{N}+\hat{j}_{0} / 2\right)^{2}\right]
\end{aligned}
$$

To construct the kernels for the integral equations (6.4) and (6.6), we basically need

$$
d t_{0} \int_{0}^{X} d j_{0} \mathcal{P}^{*}\left(j_{0}, t_{0} \mid 0\right) e^{-\bar{q}^{2} j_{0}}=2 \frac{d c}{c} \widetilde{\mathcal{P}}^{*}(\hat{q}, \hat{X}, \hat{N}),
$$

where $\hat{X}=X / c$, and where $\widetilde{\mathcal{P}}^{*}(\hat{q}, \hat{X}, \hat{N})$ is given by 


$$
\begin{aligned}
\widetilde{\mathcal{P}}^{*}(\hat{q}, \hat{X}, \hat{N})= & \sqrt{\frac{2}{\pi}} \sum_{\nu=-\infty}^{+\infty} \nu \int_{0}^{\hat{X}} d \hat{j}_{0}\left[1-4\left(\nu \hat{N}+\hat{j}_{0} / 2\right)^{2}\right] \\
& \times \exp \left[-2\left(\nu \hat{N}+\hat{j}_{0} / 2\right)^{2}-\hat{q} \hat{j}_{0}\right] \\
= & \sum_{\nu=-\infty}^{+\infty} \nu\left\{\hat { q } ^ { 2 } e ^ { 2 \nu \hat { q } \hat { N } + \hat { q } / 2 } \left[\operatorname{erfc}\left(\frac{2 \nu \hat{N}+\hat{X}+\hat{q}}{\sqrt{2}}\right)\right.\right. \\
& \left.-\operatorname{erfc}\left(\frac{2 \nu \hat{N}+\hat{q}}{\sqrt{2}}\right)\right]-\sqrt{\frac{2}{\pi}} 2 \nu \hat{N} e^{-2 \nu^{2} \hat{N}^{2}} \\
& +\sqrt{\frac{2}{\pi}}(2 \nu \hat{N}+\hat{X}-\hat{q}) \\
& \left.\times \exp \left[-\frac{(2 \nu \hat{N}+\hat{X})^{2}}{2}-\hat{q} \hat{X}\right]\right\} .
\end{aligned}
$$

In terms of $\widetilde{\mathcal{P}}^{*}(\hat{q}, \hat{X}, \hat{N})$, the kernel of Eq. (6.6) takes the form

$$
\begin{aligned}
2 \frac{d c}{c} \mathcal{K}_{E}(\hat{q}, \hat{N}) & =d t_{0} \int_{0}^{N} d j_{0} \mathcal{P}^{*}\left(j_{0}, t_{0} \mid 0\right)\left(e^{-\bar{q}^{2} j_{0}}+e^{-\bar{q}^{2}\left(N-j_{0}\right)}\right) \\
& =2 \frac{d c}{c}\left[\widetilde{\mathcal{P}}^{*}(\hat{q}, \hat{N}, \hat{N})+e^{-\hat{q} \hat{N}} \widetilde{\mathcal{P}}^{*}(-\hat{q}, \hat{N}, \hat{N})\right] .
\end{aligned}
$$

The kernel of Eq. (6.4) can be written as

$$
\begin{aligned}
& 2 \frac{d c}{\hat{q}} \mathcal{K}_{c}(\hat{q}, \hat{N}, \hat{M}) \\
& =d t_{0} \int_{0}^{N} d j_{0} \int_{(N-M) / 2}^{(N+M) / 2} d k \mathcal{P}^{*}\left(j_{0}, t_{0} \mid 0\right) e^{-\bar{q}^{2}\left|j_{0}-k\right|} \\
& =2 \frac{d c}{\hat{q}}\left\{2 \widetilde{\mathcal{P}}^{*}\left(0, \frac{\hat{N}+\hat{M}}{2}, \hat{N}\right)-2 \widetilde{\mathcal{P}}^{*}\left(0, \frac{\hat{N}-\hat{M}}{2}, \hat{N}\right)\right. \\
& -e^{\hat{q}(\hat{N}-\hat{M}) / 2}\left[\widetilde{\mathcal{P}}^{*}(\hat{q}, \hat{N}, \hat{N})-\widetilde{\mathcal{P}}^{*}\left(\hat{q}, \frac{\hat{N}-\hat{M}}{2}, \hat{N}\right)\right] \\
& +e^{-\hat{q}(\hat{N}-\hat{M}) / 2} \widetilde{\mathcal{P}}^{*}\left(-\hat{q}, \frac{\hat{N}-\hat{M}}{2}, \hat{N}\right) \\
& +e^{\hat{q}(\hat{N}+\hat{M}) / 2}\left[\widetilde{\mathcal{P}} *(\hat{q}, \hat{N}, \hat{N})-\widetilde{\mathcal{P}}^{*}\left(\hat{q}, \frac{\hat{N}+\hat{M}}{2}, \hat{N}\right)\right] \\
& \left.-e^{-\hat{q}(\hat{N}+\hat{M}) / 2} \widetilde{\mathcal{P}} *\left(-\hat{q}, \frac{\hat{N}+\hat{M}}{2}, \hat{N}\right)\right\} .
\end{aligned}
$$

\section{Discussion of the probability density of tube destruction}

Comparing the present results with those of Sec. IV C, we can verify the internal consistency of our random walk ap- proximation. From $\mathcal{P}_{\max , j}^{(T)}\left(n_{m}, n_{j}, t\right)$ [Eq. (3.20)], we can derive the probability that a part of the initial tube still exists at time $t$,

$$
\mathcal{P}^{(T)}(t)=\sum_{n_{j}=-\infty}^{-\infty} \sum_{n_{m}=0}^{N} \mathcal{P}_{\max , j}^{(T)}\left(n_{m}, n_{j}, t\right)
$$

A straightforward calculation starting from Eqs. (4.26) and (4.27) yields

$$
\mathcal{P}^{(T)}(t)=1+4 \sum_{\nu=1}^{\infty}(-1)^{\nu} \nu \operatorname{erfc}\left(\frac{\nu}{\sqrt{2}} \hat{N}\right)=\widetilde{\mathcal{P}}^{(T)}\left(\frac{N}{c}\right) .
$$

The probability that the tube is destroyed within time interval $d t_{0}$, corresponding to

$$
d c=c\left(t_{0}+d t_{0}\right)-c\left(t_{0}\right),
$$

can be calculated as $-d c(\partial / \partial c) \mathcal{P}^{(T)}$ :

$$
\begin{aligned}
-d t_{0} \frac{\partial}{\partial t_{0}} \mathcal{P}^{(T)} & =-d c \frac{\partial}{\partial c} \widetilde{\mathcal{P}}^{(T)}\left(\frac{N}{c}\right) \\
& =-4 \frac{d c}{c} \sqrt{\frac{2}{\pi}} \hat{N} \sum_{\nu=1}^{\infty}(-1)^{\nu} \nu^{2} e^{-(1 / 2) \nu^{2} \hat{N}^{2}} .
\end{aligned}
$$

On the other hand, we can calculate this probability also as

$$
2 d t_{0} \sum_{j_{0}=0}^{N} \mathcal{P}^{*}\left(j_{0}, t_{0} \mid 0\right)=4 \frac{d c}{c} \widetilde{\mathcal{P}}^{*}(0, \hat{N}, \hat{N}),
$$

where the factor of 2 takes the two chain ends into account. It is easily verified that these two expressions are identical. Thus the following relation holds:

$$
-\frac{\partial}{\partial t} \mathcal{P}^{(T)}(t)=2 \sum_{j_{0}=0}^{N} \mathcal{P}^{*}\left(j_{0}, t_{0} \mid 0\right) .
$$

This identity guarantees the validity of the normalization

$$
S_{E}(q=0, M, N) \equiv \sum_{j=(M-N) / 2}^{(M-N) / 2} 1=M+1
$$

for all times. From the definition of $S_{E}^{(T)}$, we have

$$
S_{E}^{(T)}(0, t ; M, N)=\sum_{j=(N-M) / 2}^{(N+M) / 2} \overline{\Theta\left(j_{>}-j_{<}\right)}=(M+1) \mathcal{P}^{(T)}(t) .
$$

Substituting Eqs. (6.21) and (6.23) into the integral equation (6.6), we find

$$
\begin{aligned}
S_{E}(0, t ; M, N)= & (M+1) \mathcal{P}^{(T)}(t)-\int_{0}^{t} d t_{0}\left(\frac{\partial}{\partial t_{0}} \mathcal{P}^{(T)}\left(t_{0}\right)\right) \\
& \times S_{E}\left(0, t-t_{0} ; M, N\right) .
\end{aligned}
$$




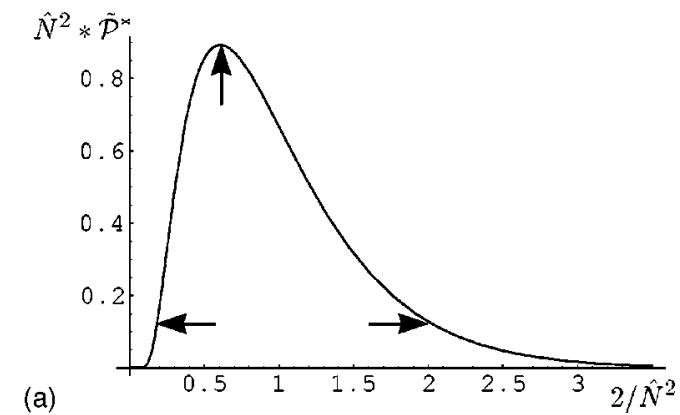

(b)

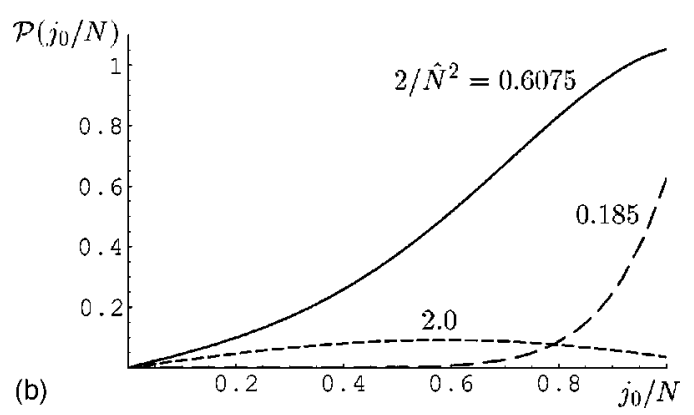

FIG. 5. Distribution functions for complete tube destruction. (a) Probability density of complete tube destruction as a function of $2 / \hat{N}^{2} \sim t / T_{3}$. Normalization, $\int_{0}^{\infty} d\left(2 / \hat{N}^{2}\right) \hat{N}^{2} \widetilde{\mathcal{P}}^{*}(0, \hat{N}, \hat{N})=1$. (b) Probability density of complete tube destruction as a function of the position $j_{0} / N$ of the final segment of the original tube, $d\left(2 / \hat{N}^{2}\right) \mathcal{P}\left(j_{0} / N\right)=d t_{0} \mathcal{P}^{*}\left(j_{0}, t_{0} \mid 0\right)$. The chain leaves the tube with end 0 . The values of $2 / \hat{N}^{2}$ chosen are indicated by arrows in (a).

Partial integration together with

$$
S_{E}(0,0 ; M, N)=M+1, \quad \mathcal{P}^{(T)}(0)=1
$$

yields

$$
0 \equiv \int_{0}^{t} d t_{0} \mathcal{P}^{(T)}\left(t_{0}\right) \frac{\partial}{\partial t_{0}} S_{E}\left(0, t-t_{0} ; M, N\right),
$$

with only the trivial solution

$$
\frac{\partial}{\partial t_{0}} S_{E}\left(0, t-t_{0} ; M, N\right) \equiv 0 .
$$

Together with Eq. (6.25), this proves Eq. (6.22). The corresponding analysis can be applied to Eq. (6.4), yielding the correct normalization

$$
S_{c}(0, t ; M, N) \equiv(M+1)^{2} .
$$

To get an impression of the time dependence of complete tube destruction, we in Fig. 5(a) show $\hat{N}^{2} \widetilde{\mathcal{P}}^{*}(0, \hat{N}, \hat{N})$ $\sim-(\partial / \partial t) \mathcal{P}^{(T)}(t)$ [cf. Eqs. (6.20) and (6.21)] as a function of $2 / \hat{N}^{2}=2(c / N)^{2}=\pi\left[\bar{l}_{s} \overline{n_{\max }(0, t) / N}\right]^{2}$. This choice of the variable is motivated by the relation $\hat{N}^{-2} \sim t / T_{3}$, cf. Eq. (7.3). As we see, noticeable tube destruction starts at $2 / \hat{N}^{2}$ $\approx 0.1$ and is essentially completed at $2 / \hat{N}^{2} \approx 3.5$. The variation of $\hat{N}^{2} \widetilde{\mathcal{P}}^{*}$ as shown here, dominates the time dependence of the kernels $(6.15),(6.16)$. It allows us to solve the integral equation (6.6) for finite time $t$ by a finite number of iterations, the result being exact within the numerical accuracy of our calculation.

To close this section, we evaluate the probability that the initial tube finally is destroyed at the position of segment $j_{0}$, with chain end 0 being the last part residing in the initial tube [Eq. (6.12)]. Figure 5(b) shows the dependence on $j_{0} / N$ for several values of $\hat{N}$. As expected, for shorter times $2 / \hat{N}^{2}<1$, chain end 0 leaves the tube close to the other end $\left(j_{0} / N \approx 1\right)$. With increasing time the most probable point of final destruction slowly shifts to the center of the tube, but for times where the rate of the tube destruction is maximal [corresponding to the maximum in Fig. 5(a)], the shape of $\mathcal{P}^{*}\left(j_{0}, t_{0} \mid 0\right)$ is still quite unsymmetric.

Obviously, the distribution functions considered here are closely related to the right-hand side of Eq. (2.9), which is determined by the part of the original tube that is still occupied at time $t$ (see Refs. $[1,12]$ ). In this context, it is interesting to note that Des Cloizeaux [16] modified the expression (2.9) by replacing $\tau=t / \tau_{d}$ in the exponent by some more complicated time dependence, meant to take the local motion near an entanglement point into account. This modification is quite similar to our introduction of the quantity $\hat{N}^{2}$ replacing $t / \tau_{d}$. We note, however, that $\hat{N}=N / c$ via the crossover behavior of $c=c(t, N)$ takes end effects such as tube length fluctuations into account rather than internal motion.

\section{THE LIMIT OF LARGE TIME AND THE PRIMITIVE CHAIN MODEL}

\section{A. Special cases}

If the time is large compared to the equilibration time $T_{2}$ of the chain, our results simplify since the parameters $a$ and $b$ can be replaced by their limiting values

$$
a=1=b \quad \text { for } \quad t \gg T_{2} .
$$

This implies that all segments experience the same curvilinear shift, which is the basic assumption of the primitive chain model. Furthermore, $A_{1}(j, t) \rightarrow \hat{t} / N$ for $t \gg T_{2}$ [cf. Eq. (3.5)], and the parameter $c[$ Eq. $(4.25)]$ reduces to

$$
\begin{aligned}
c & =\sqrt{\frac{\pi}{2}} \bar{l}_{s} \overline{n_{\max }(0, t)} \rightarrow\left(\frac{\bar{l}_{s}^{2} \rho_{0}}{2}\right)^{1 / 2} \sum_{s=1}^{t} \frac{1}{s} A_{1}^{1 / 2}(0, s) \\
& =\left(2 \frac{\bar{l}_{s}^{2} \rho_{0}}{N}\right)^{1 / 2} \hat{t}^{1 / 2} .
\end{aligned}
$$

Thus

$$
\hat{N}^{-1}=\frac{c}{N} \rightarrow\left(\frac{\hat{t}}{2 \hat{T}_{3}}\right)^{1 / 2}
$$

becomes a direct measure of $\hat{t} / \hat{T}_{3}$, where for brevity we introduced 


$$
p T_{3}=\hat{T}_{3}=\frac{N^{3}}{4 \bar{l}_{s}^{2} \rho_{0}}
$$

as measure of the reptation time. With relations (7.1), all the integrals in Eqs. (4.37)-(4.44) can be evaluated analytically, resulting in a fairly lengthy expression for $S_{c}^{(T)}(q, t ; M, N)$ as a sum of terms involving error functions and Gaussians. We here quote the result in those limits, in which $S_{c}^{(T)}$ becomes identical to the full scattering function $S_{c}$, which is the case for either short time, $t / T_{3} \ll 1$, or large wave vectors, $q^{2} R_{g}^{2}$ $\gg 1$.

\section{Limit $t / T_{3} \rightarrow 0$ with fixed $Q=q^{2} R_{g}^{2}$}

We find

$$
\frac{S_{c}(q, t ; N, N)}{N^{2}}=D(Q)-\frac{t}{2 T_{3}}\left(1-e^{-Q}\right)+O\left(\frac{t}{T_{3}}\right)^{3 / 2} .
$$

Recall that $D(Q)$ denotes the Debye function. Of course, this limit can be attained only for an extremely long chain, since relation (7.1) implies $T_{2} / T_{3} \rightarrow 0$, i.e., $N \rightarrow \infty$. The result (7.5) shows that for such a chain relaxation becomes observable only for $t \gg T_{2}$. Furthermore, with increasing $Q$, the time variation of $S_{c}$ becomes rapidly insensitive to the scattering vector.

\section{Limit $Q=q^{2} R_{g}^{2} \rightarrow \infty$ with fixed $\bar{q}^{2}$ and $t / T_{3}$}

In this limit, our result reads

$$
\begin{aligned}
\frac{S_{c}(q, t ; N, N)}{N^{2} D(Q)}= & 1-\sqrt{\frac{2}{\pi}} \frac{2}{\hat{N}}+4 \sum_{\nu=1}^{\infty}(-1)^{\nu}\left[\nu \operatorname{erfc} \frac{\nu \hat{N}}{\sqrt{2}}\right. \\
& \left.-\sqrt{\frac{2}{\pi}} \frac{1}{\hat{N}} e^{-\left(\nu^{2} / 2\right) \hat{N}^{2}}\right],
\end{aligned}
$$

which is the Poisson transform of

$$
\frac{S_{c}(q, t ; N, N)}{N^{2} D(Q)}=\frac{8}{\pi^{2}} \sum_{p=1}^{\infty}(2 p-1)^{-2} \exp \left[-(2 p-1)^{2} \frac{\pi^{2}}{2 \hat{N}^{2}}\right] .
$$

We thus recover the result of Refs. [12,13], Eq. (2.9), provided we identify

$$
\frac{t}{\tau_{d}}=\frac{\pi^{2}}{2 \hat{N}^{2}}=\frac{\pi^{2}}{4} \frac{\hat{t}}{\hat{T}_{3}}
$$

leading to

$$
p \tau_{d}=\frac{N^{3}}{\pi^{2} \bar{l}_{s}^{2} \rho_{0}}
$$

From Ref. [2], Eq. (6.19), we can take the relation of $\tau_{d}$ to the parameters of the underlying Rouse model, which in our notation reads

$$
\tau_{d}=\frac{N^{3} l_{0}^{2}}{\pi^{2} \gamma_{0} N_{e}}
$$

[Replacement $\quad \zeta \rightarrow 1 / \gamma_{0}, \quad b \rightarrow l_{0}, a^{2} \rightarrow l_{0}^{2} N_{e}, \quad k_{B} T=1 \quad$ in Ref. [2], Eq. (6.19).] Thus

$$
\frac{p l_{0}^{2}}{\gamma_{0} N_{e}}=\left(\bar{l}_{s}^{2} \rho_{0}\right)^{-1}
$$

and Eq. (5.23) results.

\section{B. Proof of asymptotic equivalence to the primitive chain model}

Having recovered the results of Doi and Edwards for $q^{2} R_{g}^{2} \gg 1, t \gg T_{2}$, we clearly may ask whether for $t \gg T_{2}$, the two approaches yield identical results irrespective of $q^{2} R_{g}^{2}$. This is not obvious since formally the approaches are quite different. Doi and Edwards [13,2] start from a diffusion equation for $S(q, t ; j, k, N)$. With the relation among model parameters established in Sec. VD, this equation takes the form

$$
\left[\frac{N}{p \bar{l}_{s}^{2} \rho_{0}} \frac{\partial}{\partial t}-\frac{\partial^{2}}{\partial j^{2}}\right] S(q, t ; j, k, N)=0
$$

This is amended by the initial condition

$$
S(q, 0 ; j, k, N)=\exp \left(-\bar{q}^{2}|j-k|\right)
$$

and boundary conditions

$$
\begin{gathered}
\lim _{j \rightarrow 0} \frac{\partial}{\partial j} S(q, t ; j, k, N)=\bar{q}^{2} S(q, t ; 0, k, N), \\
\lim _{j \rightarrow N} \frac{\partial}{\partial j} S(q, t ; j, k, N)=-\bar{q}^{2} S(q, t ; N, k, N) .
\end{gathered}
$$

On the other hand, according to our theory, $S(q, t ; j, k, N)$ obeys Eq. (6.3), written in the continuous chain model as

$$
\begin{aligned}
S(q, t ; j, k, N)= & S^{(T)}(q, t ; j, k, N)+\int_{0}^{t} d t_{0} \int_{0}^{N} d j_{0} \mathcal{P}^{*}\left(j_{0}, t_{0} \mid 0\right) \\
& \times\left\{e^{-\bar{q}^{2}\left|j_{0}-k\right|} S\left(q, t-t_{0} ; j, 0, N\right)\right. \\
& \left.+e^{-\bar{q}^{2}\left|N-j_{0}-k\right|} S\left(q, t-t_{0} ; N-j, 0, N\right)\right\} .
\end{aligned}
$$

The inhomogeneity takes the form

$$
S^{(T)}(q, t ; j, k, N)=\int_{0}^{N} d y \int_{y-N}^{y} d z \sum_{\nu} \hat{\mathcal{P}}_{\nu}(y, z) F(y, z ; j, k),
$$

where 


$$
\begin{aligned}
\hat{\mathcal{P}}_{\nu}(y, z)= & \frac{1}{\sqrt{2 \pi} c^{2}} \lim _{a \rightarrow 1} \mathcal{P}_{\max , j}^{(\nu)}\left(\frac{y}{c}, \frac{z}{c}, a\right) \\
= & \sqrt{\frac{2}{\pi}} \frac{1}{c^{3}}[(\nu+1)(2 \nu N+2 y-z) \\
& \times e^{-\left(1 / 2 c^{2}\right)(2 \nu N+2 y-z)^{2}} \\
& \left.-\nu(2 \nu N-z) e^{-\left(1 / 2 c^{2}\right)(2 \nu N-z)^{2}}\right] .
\end{aligned}
$$

The rescaling of the variables $y$ and $z$ serves to isolate the time dependence which now is contained in $c$ only [cf. Eq. (7.2)]. The function $F$ collects all contributions contained in $\mathcal{S}_{1}, \mathcal{S}_{2}, \mathcal{S}_{3}$ [Eq. (4.5)], and is found to be

$$
\begin{aligned}
F(y, z ; j, k)= & e^{-\bar{q}^{2}|k-j-z|}+\Theta(k-j-z) \Theta(y-k) \\
& \times\left[e^{-\bar{q}^{2}(2 y-z-k-j)}-e^{-\bar{q}^{2}(k-j-z)}\right] \\
& +\Theta(j+z-k) \Theta(y-j-z)\left[e^{-\bar{q}^{2}(2 y-z-k-j)}\right. \\
& \left.-e^{-\bar{q}^{2}(j-k+z)}\right]+\Theta(k-j+z) \Theta(j-z+y-N) \\
& \times\left[e^{-\bar{q}^{2}(k+j-z+2 y-2 N)}-e^{-\bar{q}^{2}(k-j+z)}\right] \\
& +\Theta(j-k-z) \Theta(k-N+y) \\
& \times\left[e^{-\bar{q}^{2}(k+j-z+2 y-2 N)}-e^{-\bar{q}^{2}(j-k-z)}\right]
\end{aligned}
$$

Here the last two contributions arise from the last two terms in Eq. (4.8), which a priori involve the distribution function

$$
\overline{\Theta\left(j_{>}-j_{<}\right) \delta_{n_{m}, n_{\max }(N, t)} \delta_{n_{j}, n(j, t)}} .
$$

Interchange of the chain ends transforms this distribution to $\mathcal{P}_{\max , j}^{(T)}\left(n_{m},-n_{j} ; t\right)[$ Eq. (3.20)] and implies that we have to take $j_{>}=N-y$ and $j(t)=j-z$ in the corresponding contributions to $F(y, z ; j, k)$.

By construction, our form of $S(q, t ; j, k, N)$ obeys the initial condition (7.10). To derive Eq. (7.9), we apply the operator

$$
\mathcal{D}=\frac{N}{p \bar{l}_{s}^{2} \rho_{0}} \frac{\partial}{\partial t}-\frac{\partial^{2}}{\partial j^{2}}
$$

to the integral equation (7.12) to find

$$
\begin{aligned}
\mathcal{D} S(q, t ; j, k, N)= & \mathcal{D} S^{(T)}(q, t ; j, k, N) \\
& +\frac{N}{p \bar{l}_{s}^{2} \rho_{0}} \int_{0}^{N} d j_{0} \mathcal{P}^{*}\left(j_{0}, t \mid 0\right)\left\{e^{-\bar{q}^{2}\left|j_{0}-k\right|-\bar{q}^{2} j}\right. \\
& \left.+e^{-\bar{q}^{2}\left|N-j_{0}-k\right|-\bar{q}^{2}(N-j)}\right\} \\
& +\int_{0}^{t} d t_{0} \int_{0}^{N} d j_{0} \mathcal{P}^{*}\left(j_{0}, t_{0} \mid 0\right) \\
& \times\left\{e^{-\bar{q}^{2}\left|j_{0}-k\right|} \mathcal{D} S\left(q, t-t_{0} ; j, 0, N\right)\right. \\
& \left.+e^{-\bar{q}^{2}\left|N-j_{0}-k\right|} \mathcal{D} S\left(q, t-t_{0} ; N-j, 0, N\right)\right\}
\end{aligned}
$$

This is an integral equation for $\mathcal{D} S$ which has only the trivial solution

$$
\mathcal{D} S(q, t ; j, k, N) \equiv 0,
$$

provided that the inhomogeneity vanishes. We first consider the contribution $\mathcal{D} S^{(T)}$ and note that in view of Eq. (7.2), $\mathcal{D}$ can be written as

$$
\mathcal{D}=\frac{1}{c} \frac{\partial}{\partial c}-\frac{\partial^{2}}{\partial j^{2}},
$$

resulting in

$$
\begin{aligned}
\mathcal{D} S^{(T)}= & \sum_{\nu} \int_{0}^{N} d y \int_{y-N}^{y} d z\left\{\left(\frac{1}{c} \frac{\partial}{\partial c} \hat{\mathcal{P}}_{\nu}(y, z)\right) F(y, z ; j, k)\right. \\
& \left.-\hat{\mathcal{P}}_{\nu}(y, z) \frac{\partial^{2}}{\partial j^{2}} F(y, z ; j, k)\right\} .
\end{aligned}
$$

$F(y, z ; j, k)[$ Eq. (7.15)] is a sum of terms that depend on $j$ and $z$ exclusively via the combinations $j+z$ or $j-z$, respectively. Thus $\partial^{2} / \partial j^{2}$ is equivalent to $\partial^{2} / \partial z^{2}$, and partial integration yields

$$
\begin{aligned}
\mathcal{D} S^{(T)}= & \sum_{\nu} \int_{0}^{N} d y \int_{y-N}^{y} d z F(y, z ; j, k)\left(\frac{1}{c} \frac{\partial}{\partial c}-\frac{\partial^{2}}{\partial z^{2}}\right) \hat{\mathcal{P}}_{\nu}(y, z) \\
& -\sum_{\nu} \int_{0}^{N} d y\left\{\left.\hat{\mathcal{P}}_{\nu}(y, y) \frac{\partial}{\partial z}\right|_{y} F(y, z ; j, k)-\left.\hat{\mathcal{P}}_{\nu}(y, y-N) \frac{\partial}{\partial z}\right|_{y-N} F(y, z ; j, k)\right. \\
& \left.-\left.F(y, y ; j, k) \frac{\partial}{\partial z}\right|_{y} \hat{\mathcal{P}}_{\nu}(y, z)+\left.F(y, y-N ; j, k) \frac{\partial}{\partial z}\right|_{y-N} \hat{\mathcal{P}}_{\nu}(y, z)\right\} .
\end{aligned}
$$


It is easily verified that in this expression the first term vanishes identically, and after some calculation exploiting relations like

$$
f(z, \nu)=(2 \nu N-z) \exp \left[-\frac{(2 \nu N-z)^{2}}{2 c^{2}}\right] \equiv f(z+2 N, \nu+1),
$$

we find

$$
\begin{aligned}
\sqrt{2 \pi} & c^{3} \mathcal{D} S^{(T)}=e^{-\bar{q}^{2} j} \mathcal{C}(k)+e^{-\bar{q}^{2}(N-j)} \mathcal{C}(N-k) \\
\mathcal{C}(k)= & 2 N \sum_{\nu}\left[4 \nu^{2} f_{0}(0, \nu) e^{-\bar{q}^{2} k}-(2 \nu-1)^{2}\right. \\
& \left.\times f_{0}(N, \nu) e^{-\bar{q}^{2}(N-k)}\right] \\
& -4 \bar{q}^{2} c^{2} \sum_{\nu} \nu\left[f_{0}(N, \nu) e^{-\bar{q}^{2}(N-k)}-2 f_{0}(k, \nu)\right] \\
& -4 \bar{q}^{4} c^{2} \int_{0}^{N} d y \sum_{\nu} \nu f_{0}(y, \nu) e^{-\bar{q}^{2}|k-y|}
\end{aligned}
$$

where

$$
f_{0}(y, \nu)=\exp \left[-\frac{(2 \nu N-y)^{2}}{2 c^{2}}\right]
$$

We now turn to the second part of the inhomogeneity in Eq. (7.17), and we use Eq. (6.12) together with $d c / d t$ $=p \bar{l}_{s}^{2} \rho_{0} /(N c)[\mathrm{cf} . \mathrm{Eq} .(7.2)]$, to write

$$
\begin{aligned}
\sqrt{2 \pi} c^{3} \frac{N}{p \bar{l}_{s}^{2} \rho_{0}} \mathcal{P}^{*}\left(j_{0}, t \mid 0\right)= & 4 \sum_{\nu} \nu\left[1-\frac{4}{c^{2}}\left(\nu N+\frac{j_{0}}{2}\right)^{2}\right] \\
& \times \exp \left[-\frac{2}{c^{2}}\left(\nu N+\frac{j_{0}}{2}\right)^{2}\right] .
\end{aligned}
$$

Thus

$$
\begin{aligned}
& \sqrt{2 \pi} c^{3} \frac{N}{p \bar{l}_{s}^{2} \rho_{0}} \int_{0}^{N} d j_{0} \mathcal{P}^{*}\left(j_{0}, t \mid 0\right)\left\{e^{-\bar{q}^{2}\left|j_{0}-k\right|-\bar{q}^{2} j}\right. \\
& \left.\quad+e^{-\bar{q}^{2}\left|N-j_{0}-k\right|-\bar{q}^{2}(N-j)}\right\} \\
& =e^{-\bar{q}^{2} j} \widetilde{\mathcal{C}}(k)+e^{-\bar{q}^{2}(N-j)} \widetilde{\mathcal{C}}(N-k)
\end{aligned}
$$

with

$$
\begin{aligned}
\widetilde{\mathcal{C}}(k)= & 4 \sum_{\nu} \nu \int_{0}^{N} d j_{0}\left[1-\frac{\left(2 \nu N+j_{0}\right)^{2}}{c^{2}}\right] \\
& \times \exp \left[-\frac{\left(2 \nu N+j_{0}\right)^{2}}{2 c^{2}}-\bar{q}^{2}\left|j_{0}-k\right|\right] .
\end{aligned}
$$

A short calculation shows that indeed

$$
\widetilde{\mathcal{C}}(k)=-\mathcal{C}(k)
$$

Thus the inhomogeneity in Eq. (7.17) vanishes and $S(q, t ; j, k, N)$ obeys the diffusion equation (7.18).

Checking the boundary conditions (7.11) is an even simpler task. Direct calculation yields

$$
\begin{aligned}
& \left.\frac{\partial}{\partial j}\right|_{0} S^{(T)}(q, t ; j, k, N) \\
& \quad=\left.\int_{0}^{N} d y \int_{y-N}^{y} d z \sum_{\nu} \hat{\mathcal{P}}_{\nu}(y, z) \frac{\partial}{\partial j}\right|_{0} F(y, z ; j, k) \\
& \quad \equiv \bar{q}^{2} S^{(T)}(q, t ; 0, k, N) \\
& \frac{\partial}{\partial j} S^{(T)}(q, t ; j, k, N) \equiv-\bar{q}^{2} S^{(T)}(q, t ; N, k, N)
\end{aligned}
$$

and differentiating the integral equation (7.12), we find

$$
\begin{aligned}
S^{\prime}(q, t ; j, k, N) \equiv & \frac{\partial}{\partial j} S(q, t ; j, k, N) \\
= & \frac{\partial}{\partial j} S^{(T)}(q, t ; j, k, N) \\
& +\int_{0}^{t} d t_{0} \int_{0}^{N} d j_{0} \mathcal{P}^{*}\left(j_{0}, t_{0} \mid 0\right) \\
& \times\left\{e^{-\bar{q}^{2}\left|j_{0}-k\right|} S^{\prime}\left(q, t-t_{0} ; j, 0, N\right)\right. \\
& \left.-e^{-\bar{q}^{2}\left|N-j_{0}-k\right|} S^{\prime}\left(q, t-t_{0}, N-j, 0, N\right)\right\} .
\end{aligned}
$$

Now writing

$$
\begin{gathered}
S^{\prime}(q, t ; 0, k, N)=\bar{q}^{2} \hat{S}_{1}(q, t, k, N), \\
S^{\prime}(q, t ; N, k, N)=-\bar{q}^{2} \hat{S}_{2}(q, t, k, N),
\end{gathered}
$$

we find from Eqs. (7.26)-(7.28),

$$
\begin{aligned}
\hat{S}_{1}(q, t, k, N)= & S^{(T)}(q, t ; 0, k, N)+\int_{0}^{t} d t_{0} \int_{0}^{N} d j_{0} \mathcal{P}^{*}\left(j_{0}, t_{0} \mid 0\right) \\
& \times\left\{e^{-\bar{q}^{2}\left|j_{0}-k\right|} \hat{S}_{1}\left(q, t-t_{0} ; 0, N\right)\right. \\
& \left.+e^{-\bar{q}^{2}\left|N-j_{0}-k\right|} \hat{S}_{2}\left(q, t-t_{0}, 0, N\right)\right\},
\end{aligned}
$$

$$
\begin{aligned}
\hat{S}_{2}(q, t, k, N)= & S^{(T)}(q, t ; N, k, N)+\int_{0}^{t} d t_{0} \int_{0}^{N} d j_{0} \mathcal{P}^{*}\left(j_{0}, t_{0} \mid 0\right) \\
& \times\left\{e^{-\bar{q}^{2}\left|j_{0}-k\right|} \hat{S}_{2}\left(q, t-t_{0} ; 0, N\right)\right. \\
& \left.+e^{-\bar{q}^{2}\left|N-j_{0}-k\right|} \hat{S}_{1}\left(q, t-t_{0}, 0, N\right)\right\} .
\end{aligned}
$$

This is exactly the system of equations obeyed by $S(q, t ; 0, k, N)$ and $S(q, t ; N, k, N)$ [cf. Eq. (6.3)]. The uniqueness of the solution together with Eq. (7.29) thus guarantees that the boundary conditions (7.11) are obeyed. 
We thus have shown that in the limit $N \rightarrow \infty$ with $t / T_{3}$ and $q^{2} R_{g}^{2}$ fixed, our theory reproduces the results of Doi and Edwards.

\section{NUMERICAL EVALUATION AND COMPARISON TO MONTE CARLO DATA}

\section{A. Technicalities of solving the integral equations}

As shown by relations like Eqs. (6.19) and (6.20), the natural measure of time in our theory is the parameter $c$ $=c(t)$. It measures the motion of the chain ends, i.e., the time dependence of tube destruction, and is defined by Eqs. (4.25) and (3.16). In evaluating the theory, we therefore replace time by the variable

$$
x=\mathcal{X}(t)=\frac{c(t, N)}{N}=\frac{1}{\hat{N}} .
$$

Using Eq. (6.15), we write the integral equation (6.6) in the form

$$
\begin{aligned}
\bar{S}_{E}(x)= & \bar{S}_{E}^{(T)}(x)+2 \int_{0}^{x} \frac{d x^{\prime}}{x^{\prime}} \mathcal{K}_{E}\left(\bar{q}^{2} N x^{\prime}, \frac{1}{x^{\prime}}\right) \\
& \times \bar{S}_{E}\left[\mathcal{X}\left(\tau(x)-\tau\left(x^{\prime}\right)\right)\right]
\end{aligned}
$$

where $t=\tau(x)$ is the inverse function to $x=\mathcal{X}(t) . \bar{S}_{E}(x)$ and $\bar{S}_{E}^{(T)}(x)$ denote the scattering functions $S_{E}$ and $S_{E}^{(T)}$, normalized with the static coherent structure function, e.g.,

$$
\bar{S}_{E}(x)=\frac{S_{E}(q, t ; M, N)}{S_{c}(q, t=0 ; M, M)} .
$$

With corresponding notation, Eqs. (6.4) and (6.16) yield for the normalized coherent structure function

$$
\begin{aligned}
\bar{S}_{c}(x)= & \bar{S}_{c}^{(T)}(x)+\frac{4}{\bar{q}^{2}} \int_{0}^{x} \frac{d x^{\prime}}{x^{\prime}} \mathcal{K}_{c}\left(\bar{q}^{2} N x^{\prime}, \frac{1}{x^{\prime}}, \frac{M}{x^{\prime} N}\right) \\
& \times \bar{S}_{E}\left[\mathcal{X}\left(\tau(x)-\tau\left(x^{\prime}\right)\right)\right] .
\end{aligned}
$$

In Eqs. (8.2) and (8.4), we then transform from variables $x^{\prime}$ to $\hat{x}=\mathcal{X}\left(\tau(x)-\tau\left(x^{\prime}\right)\right)$ to find equations of standard Volterra form, which are solved by discretizing $x, \hat{x}$ and iteration. We note that both $\bar{S}_{E}^{(T)}(x)$ and $\bar{S}_{c}^{(T)}(x)$ for $x>2$ are negligibly small, less than $10^{-7}$, to be compared to the normalization $\bar{S}_{c}(0)=1$. Also the kernels $\mathcal{K}_{E}, \mathcal{K}_{c}$ exceed $10^{-7}$ only in the interval $0.1<x^{\prime}<2.2$. This allows for an accurate evaluation, simply using computer algebra. The numerical uncertainty of our final results is less than $0.5 \%$. In all our analysis, we used the same parameter values as in Ref. [9]. Specifically, $\bar{l}_{s}^{2} \rho_{0}$ $=1.23, p=1 / 5$, and $\bar{l}_{s}=2.364$. (We recall that the precise values of $p$ and $\bar{l}_{s}$ in fact are irrelevant for our numerical results.)
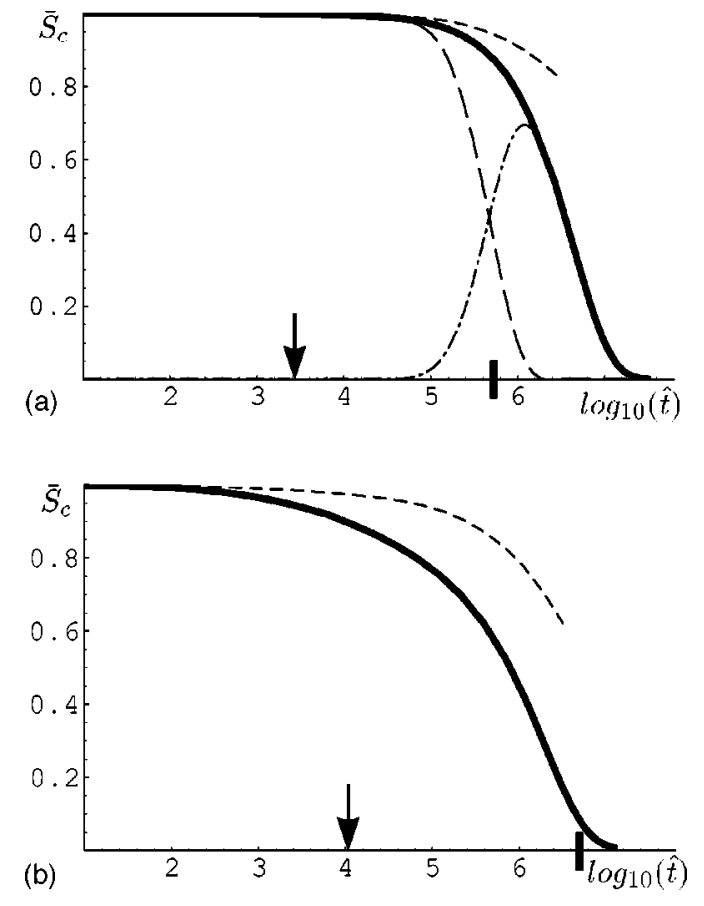

FIG. 6. Normalized coherent scattering function in different wave number regions as a function of $\log _{10}(\hat{t})$. (a) $q^{2}=0.01, N$ $=M=157$; (b) $q^{2}=1.0, N=M=317$. The thick solid lines give the full functions $\bar{S}_{c}$. Long dashes represent $\bar{S}_{c}^{(T)}$, which in (b) coincides with $\bar{S}_{c}$. Short dashes are the results neglecting all end effects. The dot-dashed line in (a) is the contribution of complete tube destruction. See the text for further explanation.

\section{B. Typical results}

We first want to illustrate the magnitude of the different contributions to the normalized structure function $\bar{S}_{c}$. Figure 6 shows results for two very different values of wave number: $q^{2} R_{g}^{2} \approx 0.27$ in Fig. 6(a), and $q^{2} R_{g}^{2} \approx 53$ in Fig. 6(b). The thick lines give the full results for $\bar{S}_{c}$, including end effects and tube destruction. Long dashes represent $\bar{S}_{c}^{(T)}$, i.e., the contribution without complete tube destruction. Short dashes represent the (normalized) contribution $\overline{\mathcal{S}}_{1}$ [Eq. (4.33)], which omits all end effects and treats the chain as embedded in an infinitely long tube. The arrows point to the internal equilibration time $\hat{T}_{2}$, defined by Eq. (5.26),

$$
\hat{T}_{2}=\frac{(N+1)^{2}}{\pi^{2}} .
$$

Finally, the heavy slashes in the time axes give the reptation time defined as the first moment of the time dependent probability density of complete tube destruction,

$$
\hat{T}_{3}=p \int_{0}^{\infty} d t_{0} t_{0}\left(-\frac{\partial}{\partial t_{0}}\right) \mathcal{P}^{(T)}\left(t_{0}\right) .
$$

Here we use Eq. (6.19) for $\partial \mathcal{P}^{(T)} / \partial\left(t_{0}\right)$. For long chains, the thus defined reptation time tends to the value given in Eq. (7.4). 


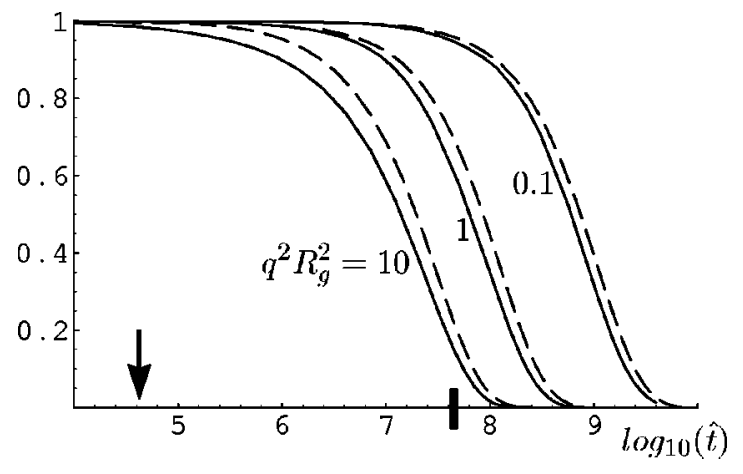

FIG. 7. Normalized coherent structure function $\bar{S}_{c}$ for $N=637$ and the values of $q^{2} R_{g}^{2}$ as indicated (solid lines). The dashed lines denote $\bar{S}_{D E}$. Arrow and slash indicate $\hat{T}_{2}$ or $\hat{T}_{3}$, respectively.

Figure 6(a) shows the typical behavior of $\bar{S}_{c}(q, t, N)$ for wave numbers that are too small to resolve the internal structure of the tube. $\bar{S}_{c}^{(T)}$ stays close to 1 up to times of order $\hat{T}_{3}$ and then rapidly drops to zero. The contribution of processes with complete tube destruction [dot-dashed line in Fig. 6(a)] is very important, and end effects become visible only at a time where also tube destruction plays a role. Note that according to Eq. (6.4), the total structure function $\bar{S}_{c}$ is a sum of two independently calculated terms: $\bar{S}_{c}^{(T)}$ (long-dashed line) and the contribution of complete tube destruction (dotdashed line). These terms add up to a smooth curve (thick solid line), an observation that demonstrates the consistency of our approach on the quantitative level. It is only for very short chains, $N \lesssim 30$, that these two contributions do not quite match. We trace this back to our approximate calculation of the kernel $\mathcal{K}_{c}$. For such short chains, tube length fluctuations and internal relaxation presumably play a role also for the kernels.

The limit of large wave vectors is illustrated with Fig. 6(b). Here configurations where the original tube has been destroyed, essentially do not contribute to the scattering. Indeed, in Fig. 6(b), the curves for $\bar{S}_{c}$ and $\bar{S}_{c}^{(T)}$ fall right on top of each other. However, end effects such as tube length fluctuations have a strong influence, as shown by the deviations among the full line and the dashed line representing $\overline{\mathcal{S}}_{1}$. They lead to a gradual decrease of $\bar{S}_{c}$, starting long before complete tube destruction becomes effective.

In the preceding section, we have shown that our theory asymptotically reduces to the primitive chain model of Doi and Edwards. To test the range of validity of the asymptotic result, we have evaluated our theory for the fairly large value $N=637$ of the chain length (corresponding to a Monte Carlo chain of 640 beads, cf. Ref. [9], Sec. II C). Figure 7 shows the results for the normalized coherent structure function $\bar{S}_{c}$ as function of $\log _{10} \hat{t}$ for a set of wave vectors: $q^{2} R_{g}^{2}=0.1$, 1.0, and 10. The dashed lines give the asymptotic result (2.5),(2.6), where we used Eq. (7.8) for $\tau_{d}$. Obviously, the time scales do not quite match: even for this long chain, the reptation time does not yet follow the $N^{3}$ law. A shift of $\log _{10} \hat{t}$ by -0.1 , equivalent to a decrease of the time scale by $20 \%$, for small $q$, such that $q^{2} R_{g}^{2} \lesssim 1$, brings the asymptotic

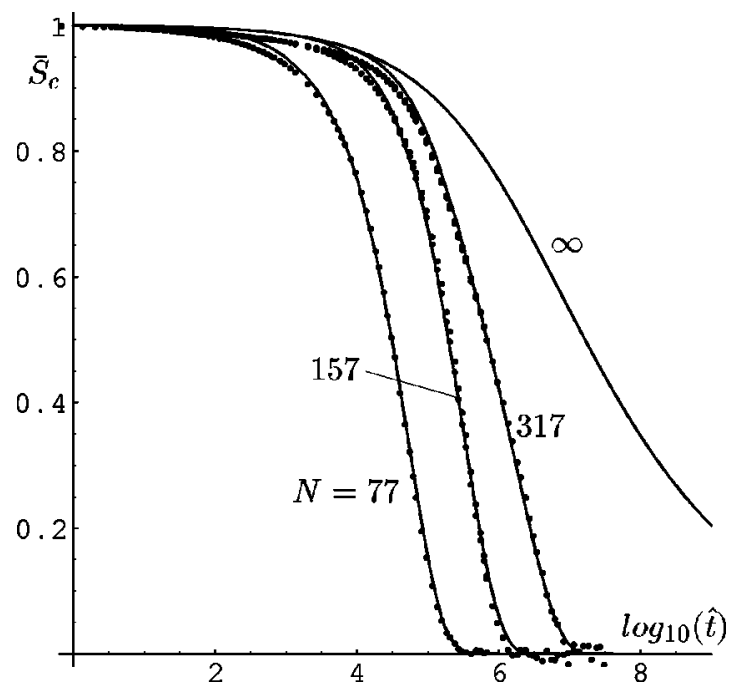

FIG. 8. Normalized coherent structure function $\bar{S}_{c}$ of a subchain of about $M=80$ beads in chains of the total length $N$ as indicated in the figure. Wave number $q=0.5$. Solid line, theory. Data points result from a simulation of the Evans-Edwards model.

results close to the results of our full model. For $q^{2} R_{g}^{2}=10$, however, even with such a shift, there remains a definite difference: the result of the full theory initially decreases faster and approaches the shifted asymptotic curve only for $\hat{t} \gtrsim 10^{2} \hat{T}_{2}$. This is an effect of internal relaxation and tube length fluctuations. The absence of a visible mismatch in the shape of the curves for smaller $q$ values just implies that with such small values again the structure of the tube cannot be resolved.

To examine more closely the influence of internal relaxation and tube length fluctuation, we in Figs. 8 and 9 show results for the scattering from internal pieces of a chain. Figure 8 shows results for $q=0.5$ and a subchain of about 80

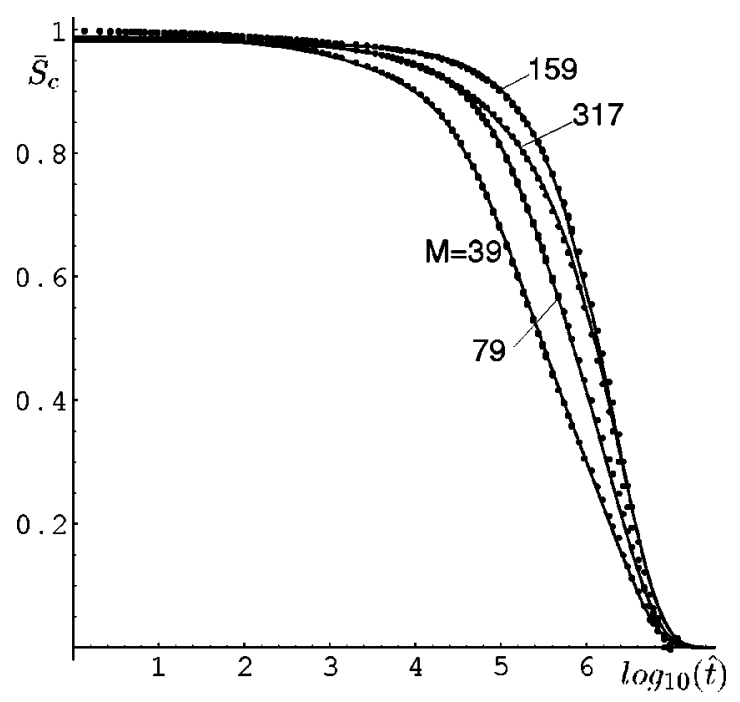

FIG. 9. Results for $\bar{S}_{c}, q=0.5, N=317$. Theoretical results (solid lines) for central subchains of lengths $M=39,79$, and 159 are compared to simulations (dots). Results for the total chain $(M=317)$ are also shown. 
beads, corresponding to $q^{2} R_{g}^{2} \approx 3.29$. The subchain is embedded as central piece in chains of different lengths, precisely: ( $N=77$, total chain), $(N=157, M=79),(N=317, M=79)$, $(N=\infty, M=79)$. This figure illustrates the suppression of end effects with increasing $N$. The asymptotic result $N=\infty$ is due to internal relaxation only. For finite $N$, the curves start to deviate from the asymptotic form as soon as wiggles created at a chain end have a non-negligible probability to reach the central piece. The characteristic time for this process scales with $(N-M)^{2}$. Figure 9 shows results for $q=0.5, N$ $=317$, and central pieces of lengths $M=317$ to $M=39$. Due to tube length fluctuations, the normalized scattering function of the total chain initially decreases faster than the result for $M=159$. Tube destruction on the average reaches the subchains at times between $\hat{t} \approx 10^{5.8}(M=159)$ or $\hat{t}$ $\approx 10^{6.4}(M=39)$, so that for large time regimes, the results for the subchains are not affected by tube length fluctuations. The decrease of the normalized scattering intensity with decreasing length of the subchain rather is due to the fact that a shorter subchain leaves its initial position in the tube earlier, i.e., it is due to internal relaxation.

In Figs. 8 and 9, we included results from a simulation of the Evans-Edwards model [17]. This model takes the chain configuration as a random walk on a cubic lattice and allows only moves of "hairpin" configurations $\mathbf{r}_{j+1}-\mathbf{r}_{j}=\mathbf{r}_{j-1}-\mathbf{r}_{j}$ as internal motion. An illustration for a two-dimensional system is shown in Fig. 1. We used the same implementation of the model as in our previous work [9], to which we refer for details. In comparing theory and simulations therefore all parameters are fixed by our previous analysis of segment motion. Since, however, the new simulations lead to better statistics, we allow for some readjustment of the relation among $\hat{t}$ and the Monte Carlo time scale: $\hat{t}=6.8 \times 10^{-2} t_{M C}$ instead of $\hat{t}=6.09 \times 10^{-2} t_{M C}$ taken previously. This yields a shift of -0.048 of the logarithmic time scale and leaves our previous results essentially unaffected.

As shown in Fig. 8, our theory in all details reproduces the time variations of the data, but the data systematically lie somewhat below the theoretical curves. This is not due to our approximations that essentially only concern the treatment of tube length fluctuations. Considering for instance the data for $N=317$, we note that the deviations from the theory are strongest for $\hat{t} \lesssim 10^{5}$, where tube destruction and tube length fluctuations are irrelevant and our theory for the internal part $M=79$ is an exact evaluation of the reptation model. Furthermore, the deviations are fairly independent of the lengths of the end pieces $(N-M) / 2$. This suggests that we see some (nonuniversal) relaxation of the microstructure. Clearly, particle hopping, which is the elementary dynamics of the reptation model, is no faithful representation of the Monte Carlo hairpin dynamics on the microscopic level. The wave vector $|\mathbf{q}|=0.5$ is large enough to resolve such details. Since the dynamic effects of microstructure should saturate at larger times, this suggests that we should scale down the theoretical curves by some factor $B_{R}<1$. This was done in Fig. 9, where $B_{R}$ ranges from 0.981 to 0.990 , depending on $M$. For $\hat{t}$ $\geq 10^{3}$, theory and data agree excellently. The same level of agreement can be reached for the data of Fig. 8. We therefore believe that our theory adequately describes the universal part of the coherent scattering function, including tube length fluctuations and (universal) internal relaxation.

A more extensive presentation of simulation results, comparing with the present and previous theories, will be published elsewhere. Here we only note that we have taken data for values of $|\mathbf{q}|$ ranging from 1.0 to 0.1 , and the $q$ dependence found for the initial deviation among theory and simulations strongly supports the interpretation as microstructure effects.

\section{SUMMARY AND CONCLUSIONS}

In this work, we have exploited the pure reptation model to calculate the coherent structure function $S_{c}(q, t ; M, N)$ of a flexible chain moving through an array of impenetrable topological obstacles. Our analysis is rigorous for a subchain (length $M$ ) in the interior of an infinitely long chain $(N$ $\rightarrow \infty$ ). This allows for a detailed comparison with an approach where the interior motion of the chain is modeled as one-dimensional Rouse motion along a coiled tube. We found that the latter model starts from unphysical nonequilibrium initial conditions, which relax only on the scale of the Rouse time $T_{2}(M)$ of the subchain. This relaxation completely distorts the time dependence of $S_{c}(q, t ; M, N=\infty)$ for times $t \lesssim T_{2}(M)$. Only for times $t \gg T_{2}(M)$, this model is equivalent to the reptation model. If applied to the total chain, "local relaxation" calculated as Rouse motion in a tube therefore is unphysical. A realistic system may show some relaxation that is specific to the microscopic dynamics, and which is not contained in the pure reptation model. However, our analysis sheds strong doubts on an interpretation of such nonuniversal effects within the framework of the model of a Rouse chain in a tube.

To evaluate the total structure function for all times, we have derived integral equations that split $S_{c}$ into a contribution $S_{c}^{(T)}$ of configurations where some part of the initial tube still exists, and the remainder. The kernel and in particular the inhomogeneities (like $S_{c}^{(T)}$ ) of these equations cannot be calculated rigorously. They involve distribution functions coupling the motion of a given segment to tube renewal, which is a non-Markovian process with memory time of the order of the Rouse time $T_{2}(N)$. To calculate the functional form of these distributions, we used a random walk approximation. We thus at each instant of time replaced the correlated process by an uncorrelated process which as closely as possible reproduces the instantaneous distributions of the correlated process. This "mean hopping rate" approximation introduces functions $c=c(t), a=a(j, t)$, and $b=b(j, t)$, which appear as parameters in the distribution functions and can be calculated from the microscopic hopping process of spared length in the reptation model. They also have a simple physical meaning: $c(t)$ measures the average extent of tube destruction, $a(j, t)$ describes the coupling of motion of segment $j$ to tube destruction, and $b(j, t)$ takes care of the inhomogeneity of the effective segment mobility along the chain, which arises from the fact that the mobile units, i.e., the wiggles of spared length, can be created and destroyed only at the chain ends. 
In the limit of long chains and time $t \gg T_{2}$, the parameters $a$ and $b$ tend to 1 irrespective of $j$, and $c \sim t^{1 / 2}$. Our theory then reproduces the results of the "primitive chain" model. Our proof of this asymptotic result amounts to a derivation of the primitive chain model from microscopic reptation dynamics. Combined with the analysis of the internal motion for $t \ll T_{2}$, this result allows for a mapping of the microscopic parameters of our reptation model to the more commonly used Rouse-type parameters.

The parameter functions $a(j, t), b(j, t)$, and $c(t)$ approach their asymptotic behavior only slowly, and it needs chain lengths of order $N / N_{e} \gtrless 300$ to find a time region where the primitive chain model is valid. In particular, for shorter chains the reptation time, if extracted by fitting the Doi-Edwards result for the primitive chain model to the large time behavior of $S_{c}(q, t, N)$ in the reptation model, does not obey the asymptotic power law $T_{3} \sim N^{3}$. As will be shown in Ref. [14], it rather exhibits the well known behavior $T_{3}$ $\sim N^{z_{\text {eff }}}$, with an effective exponent $z_{\text {eff }}>3$. The deviation of $a(j, t), b(j, t)$, and $c(t)$ from their asymptotic primitive chain behavior incorporates the effect of internal relaxation and tube length fluctuations. Our numerical evaluation of the full theory illustrates that these effects in general are quite important. In particular, we find a clear difference among the time variation of scattering from the total chain compared to scattering from internal pieces. The latter are less influenced by tube length fluctuations but are more strongly affected by internal relaxation. This leads to a peculiar behavior of $S_{c}(q, t ; M, N)$ with varying length $M$ of the internal piece, as shown in Fig. 9. Quite generally, for the total chain ( $M$ $=N$ ) it is the tube length fluctuations, that determine $S_{c}$ for times up to $t \approx 10 T_{2}$.

All our quantitative numerical results are well supported by simulations of pure reptation, exploiting the lattice model of Evans and Edwards. In view of the unavoidable approximations inherent in the theory, the quantitative agreement is quite remarkable. It suggests that our mean hopping rate approximation adequately takes care of the coupling among internal relaxation, tube length fluctuations, and global creep. A more extensive comparison to Monte Carlo data including a numerical parametrization of our analytical results will be published elsewhere [14].

\section{ACKNOWLEDGMENTS}

This work was supported by the Deutsche Forschungsgemeinschaft, SFB 237.

\section{APPENDIX A: RANDOM WALK MODEL FOR DISTRIBUTION FUNCTIONS}

1. The function $\mathcal{P}_{\max , 0}^{(T)}\left(n_{m}, n ; t\right)$

As explained in Sec. IV C, we consider a random walk $n^{\prime}(s)$ on the integer numbers, with hopping rate $p^{\prime}$. The walk starts at $n^{\prime}(0)=0$ and ends at $n^{\prime}(t)=n$. It is restricted to the interval $\left[n_{m}-N^{\prime}+1, n_{m}\right]$, with absorbing boundary conditions. To simplify the notation, we shift the interval by $N^{\prime}-n_{m}$ to

$$
\mathcal{I}=\left[1, N^{\prime}\right],
$$

with the starting point of the walk $n^{\prime}(0)=N^{\prime}-n_{m}$, and the endpoint $n^{\prime}(t)=n+N^{\prime}-n_{m}$. The hopping matrix of the walk takes the form

$$
\begin{gathered}
\hat{W}\left(N^{\prime}\right)_{j, j^{\prime}}=\left(1-2 p^{\prime}\right) \delta_{j j^{\prime}}+p^{\prime}\left(\delta_{j, j^{\prime}+1}+\delta_{j^{\prime}, j-1}\right), \\
j, j^{\prime} \in \mathcal{I} .
\end{gathered}
$$

$\mathcal{P}_{\text {max }, 0}^{(T)}\left(n_{m}, n ; t\right)$ gives the weight of the walk under the constraint that $n^{\prime}(s)=n_{m}$ is attained for at least one $s \in[0, t]$. It is easily found as

$$
\begin{aligned}
\mathcal{P}_{\max , 0}^{(T)}\left(n_{m}, n ; t\right)= & \Theta\left(n_{m}-\frac{|n|+n}{2}\right) \\
& \times \Theta\left(\frac{n-|n|}{2}-n_{m}+N^{\prime}-1\right) \\
& \times\left\{\left(\hat{W}^{t}\left(N^{\prime}\right)\right)_{1+n_{m}-n, 1+n_{m}}-\left(1-\delta_{n_{m}, 0}\right)\right. \\
& \left.\times\left(\hat{W}^{t}\left(N^{\prime}-1\right)\right)_{n_{m}-n, n_{m}}\right\} .
\end{aligned}
$$

$\hat{W}^{t}\left(N^{\prime}\right)$ can be written as

$$
\begin{aligned}
\left(\hat{W}^{t}\left(N^{\prime}\right)\right)_{j, j^{\prime}}= & \frac{2}{N^{\prime}+1} \sum_{\kappa=1}^{N^{\prime}} \sin \frac{\pi \kappa j}{N^{\prime}+1} \sin \frac{\pi \kappa j^{\prime}}{N^{\prime}+1} \\
& \times\left(1-4 p^{\prime} \sin ^{2} \frac{\pi \kappa}{2\left(N^{\prime}+1\right)}\right)^{t} .
\end{aligned}
$$

For $t \gg 1$, the last factor can be replaced by $\exp \left\{-p^{\prime} t\left[\pi^{2} \kappa^{2} /\left(N^{\prime}+1\right)^{2}\right]\right\}$, and a little calculation yields

$$
\begin{aligned}
\mathcal{P}_{\max , 0}^{(T)}\left(n_{m}, n, t\right) \approx & \Theta\left(n_{m}-\frac{|n|+n}{2}\right) \Theta\left(\frac{n-|n|}{2}-n_{m}+N^{\prime}-1\right) \\
& \times\left\{\frac{1}{N^{\prime}+1} \sum_{\kappa=1}^{\infty} e^{-p^{\prime} t\left[\pi^{2} \kappa^{2} /\left(N^{\prime}+1\right)^{2}\right]}\right. \\
& \times\left[\cos \frac{\pi \kappa n}{N^{\prime}+1}-\cos \frac{\pi \kappa\left(2 n_{m}+2-n\right)}{N^{\prime}+1}\right] \\
& -\frac{1}{N^{\prime}} \sum_{\kappa=1}^{\infty} e^{-p^{\prime} t\left(\pi^{2} \kappa^{2} / N^{\prime 2}\right)} \\
& \left.\left.\times\left[\cos \frac{\pi \kappa n}{N^{\prime}}-\cos \frac{\pi \kappa\left(2 n_{m}-n\right)}{N^{\prime}}\right]\right\} . \quad \text { (A5 }\right)
\end{aligned}
$$

In extending the sums to infinity, we neglect terms of order $\exp \left(-\pi^{2} p^{\prime} t\right)$.

Now we note that $\mathcal{P}_{\max , 0}^{(T)}$ rapidly decreases for $t>T_{3}$. As mentioned in Sec. III B, the effective hopping rate for $t$ $\sim T_{3} \gg T_{2}$ behaves as $p^{\prime} \sim 1 / N \sim 1 / N^{\prime}$, and as a consequence, the argument of the exponential in Eq. (A5) takes the form - const $\kappa^{2} t / T_{3}$. Thus $\mathcal{P}_{\max , 0}^{(T)}$ yields a relevant contribution only for smaller times: $t \leqslant T_{3}$, and for treating this time 
range, it is preferable to replace the summations in Eq. (A5) by their Poisson transform. This yields

$$
\begin{aligned}
\mathcal{P}_{\max , 0}^{(T)}\left(n_{m}, n ; t\right)= & \Theta\left(n_{m}+\frac{|n|+n}{2}\right) \Theta\left(\frac{n-|n|}{2}-n_{m}+N^{\prime}-1\right) \\
& \times \frac{1}{\sqrt{4 \pi p^{\prime} t}} \sum_{\nu=-\infty}^{+\infty}\left\{\exp \left\{-\frac{1}{p^{\prime} t}\left(\nu N^{\prime}+\frac{n}{2}\right)^{2}\right\}\right. \\
& \times\left[\exp \left\{-\frac{2 \nu}{p^{\prime} t}\left(\nu N^{\prime}+\frac{n}{2}\right)-\frac{\nu^{2}}{p^{\prime} t}\right\}-1\right] \\
& -\exp \left\{-\frac{1}{p^{\prime} t}\left(\nu N^{\prime}+n_{m}-\frac{n}{2}\right)^{2}\right\} \\
& \times\left[\operatorname { e x p } \left\{-\frac{2 \nu+2}{p^{\prime} t}\left(\nu N^{\prime}+n_{m}-\frac{n}{2}\right)\right.\right. \\
& \left.\left.\left.-\frac{(\nu+1)^{2}}{p^{\prime} t}\right\}-1\right]\right\} .
\end{aligned}
$$

Now the Gaussian prefactors of the square brackets allow for an essential contribution only for $p^{\prime} t \geqq N^{\prime 2}$, and in this region, the square brackets can be replaced by the linear approximation

$$
\begin{aligned}
\exp \left[-\frac{2 \nu}{p^{\prime} t}\left(\nu N^{\prime}+\frac{n}{2}\right)-\frac{\nu^{2}}{p^{\prime} t}\right]-1 \\
\approx-\frac{2 \nu}{p^{\prime} t}\left(\nu N^{\prime}+\frac{n}{2}\right) \exp \left[-\frac{2 \nu+2}{p^{\prime} t}\left(\nu N^{\prime}+n_{m}-\frac{n}{2}\right)\right. \\
\left.\quad-\frac{(\nu+1)^{2}}{p^{\prime} t}\right]-1 \\
\approx-\frac{2(\nu+1)}{p^{\prime} t}\left(\nu N^{\prime}+n_{m}-\frac{n}{2}\right) .
\end{aligned}
$$

Equation (4.20) from Sec. IV C is the result, which is correct to leading order in $1 / N^{\prime}$. The neglect of $1 / N^{\prime}$ corrections is consistent with treating segment indices as continuous.

\section{The function $\mathcal{P}^{*}\left(j_{0}, t_{0} \mid \mathbf{0}\right)$}

By definition, $\mathcal{P}^{*}\left(j_{0}, t_{0} \mid 0\right)$ gives the probability that the initial tube is destroyed completely at time step $t_{0}$, with $j_{0}$ being the last point, occupied by chain end 0 (see Sec. VI A). In the random walk model, this probability is given by the weight of a walk starting at $n^{\prime}(0)=0$ and ending at $n^{\prime}\left(t_{0}\right)$ $=j_{0}^{\prime}=j_{0} / l_{s}$. The point $j_{0}^{\prime}$ is reached at $t=t_{0}$ for the first time, but the point $j_{0}^{\prime}-N^{\prime}+1$ is attained for some $s \in\left[0, t_{0}\right.$ $-1]$. The walk is restricted to the interval $\left[j_{0}^{\prime}-N^{\prime}+1, j_{0}^{\prime}\right]$. Shifting the interval by $N^{\prime}-j_{0}^{\prime}$, we can express this weight as

$$
\begin{aligned}
\mathcal{P}^{*}\left(j_{0}, t_{0} \mid 0\right)= & p^{\prime}\left\{\left[\hat{W}^{t_{0}-1}\left(N^{\prime}-1\right)\right]_{N^{\prime}-1, N^{\prime}-j_{0}^{\prime}}\right. \\
& \left.-\left[\hat{W}^{t_{0}-1}\left(N^{\prime}-2\right)\right]_{N^{\prime}-2, N^{\prime}-j_{0}^{\prime}-1}\right\},
\end{aligned}
$$

where the prefactor $p^{\prime}$ gives the probability of the last step, leading from $N^{\prime}-1$ to $N^{\prime}$ (in the shifted walk). Using the explicit expression (A4) for $\hat{W}^{t}$ and exploiting $t=t_{0}-1 \gg 1$, we find

$$
\begin{aligned}
\mathcal{P}^{*}\left(j_{0}, t_{0} \mid 0\right)= & \frac{p^{\prime}}{N^{\prime}} \sum_{\kappa=1}^{\infty}\left(\cos \frac{\pi \kappa\left(j_{0}^{\prime}-1\right)}{N^{\prime}}\right. \\
& \left.-\cos \frac{\pi \kappa\left(j_{0}^{\prime}+1\right)}{N^{\prime}}\right) e^{-p^{\prime}\left(t_{0}-1\right)\left(\pi^{2} \kappa^{2} / N^{\prime 2}\right)} \\
& -\frac{p^{\prime}}{N^{\prime}-1} \sum_{\kappa=1}^{\infty}\left(\cos \frac{\pi \kappa\left(j_{0}^{\prime}-1\right)}{N^{\prime}-1}\right. \\
& \left.-\cos \frac{\pi \kappa\left(j_{0}^{\prime}+1\right)}{N^{\prime}-1}\right) e^{-p^{\prime}\left(t_{0}-1\right)\left[\pi^{2} \kappa^{2} /\left(N^{\prime}-1\right)^{2}\right]}
\end{aligned}
$$

correct up to exponentially small terms [cf. Eq. (A5)]. The Poisson transform yields

$$
\begin{aligned}
\mathcal{P}^{*}\left(j_{0}, t_{0} \mid 0\right)= & \frac{p^{\prime}}{\sqrt{4 \pi p^{\prime}\left(t_{0}-1\right)}} \\
& \times \sum_{\nu=-\infty}^{+\infty} \exp \left[-\frac{1}{p^{\prime}\left(t_{0}-1\right)}\left(\nu N^{\prime}+\frac{j_{0}^{\prime}}{2}\right)^{2}\right] \\
& \times\left\{\exp \left[\frac{1}{p^{\prime}\left(t_{0}-1\right)}\left(\nu N^{\prime}+\frac{j_{0}^{\prime}}{2}-\frac{1}{4}\right)\right]\right. \\
& -\exp \left[\frac{-1}{p^{\prime}\left(t_{0}-1\right)}\left(\nu N^{\prime}+\frac{j_{0}^{\prime}}{2}+\frac{1}{4}\right)\right] \\
& -\exp \left[\frac { 1 } { p ^ { \prime } ( t _ { 0 } - 1 ) } \left\{2 \nu\left(\nu N^{\prime}+\frac{j_{0}^{\prime}}{2}-\frac{1}{2}\right)+\nu N^{\prime}\right.\right. \\
& \left.\left.+\frac{j_{0}^{\prime}}{2}-\frac{1}{4}-\nu^{2}\right\}\right]+\exp \left[\frac { 1 } { p ^ { \prime } ( t _ { 0 } - 1 ) } \left(2 \nu \left(\nu N^{\prime}\right.\right.\right. \\
& \left.\left.\left.\left.+\frac{j_{0}^{\prime}}{2}+\frac{1}{2}\right)-\nu N^{\prime}-\frac{j_{0}^{\prime}}{2}-\frac{1}{4}-\nu^{2}\right)\right]\right\} . \quad(\mathrm{A} 9)
\end{aligned}
$$

As in Eq. (A6), we can expand in the square brackets, keeping the first nonvanishing terms which here are of second order. Identifying now $N^{\prime}=N / \bar{l}_{s}$ and $j_{0}^{\prime}=j_{0} / \bar{l}_{s}$, Eq. (6.11) in Sec. VIC results. 


\section{APPENDIX B: EXPRESSION FOR $\mathcal{P}_{j}^{(T)}\left(n_{j} ; t\right)$}

According to Eqs. (4.28) and (4.30), $\mathcal{P}_{j}^{(T)}\left(n_{j} ; t\right)$ can be determined by integrating $\mathcal{P}_{\max , j}^{(T)}$ over $n_{m}$ (or $y$, equivalently). This integral can be carried through analytically to yield

$$
\begin{aligned}
\mathcal{P}_{j}^{(\nu)}(z, a)= & \exp \left(-\frac{(z-2 a \nu \hat{N})^{2}}{2}\right)\left\{2\left(2-a^{2}\right) \nu \operatorname{erfc}\left(\frac{a z+a_{2} \nu \hat{N}}{\sqrt{a_{2}}}\right)-\left(\left(2-a^{2}\right) \nu+\frac{1}{2}\right) \operatorname{erfc}\left(\frac{a z+a_{2} \nu \hat{N}+\hat{N}}{\sqrt{a_{2}}}\right)-\left(\left(2-a^{2}\right) \nu-\frac{1}{2}\right)\right. \\
& \times \operatorname{erfc}\left(\frac{a z+a_{2} \nu \hat{N}-\hat{N}}{\sqrt{a_{2}}}\right)+\nu a(z-2 \nu a \hat{N})\left[2\left(a z+a_{2} \nu \hat{N}\right) \operatorname{erfc}\left(\frac{a z+a_{2} \nu \hat{N}}{\sqrt{a_{2}}}\right)-\left(a z+a_{2} \nu \hat{N}+\hat{N}\right)\right. \\
& \left.\times \operatorname{erfc}\left(\frac{a z+a_{2} \nu \hat{N}+\hat{N}}{\sqrt{a_{2}}}\right)-\left(a z+a_{2} \nu \hat{N}-\hat{N}\right) \operatorname{erfc}\left(\frac{a z+a_{2} \nu \hat{N}-\hat{N}}{\sqrt{a_{2}}}\right)\right] \\
& \left.-\sqrt{\frac{a_{2}}{\pi}} \nu a(z-2 \nu a \hat{N})\left[2 \exp \left(-\frac{\left(a z+a_{2} \nu \hat{N}\right)^{2}}{a_{2}}\right)-\exp \left(-\frac{\left(a z+a_{2} \nu \hat{N}+\hat{N}\right)^{2}}{a_{2}}\right)-\exp \left(-\frac{\left(a z+a_{2} \nu \hat{N}-\hat{N}\right)^{2}}{a_{2}}\right)\right]\right\},
\end{aligned}
$$

with $a_{2}=2\left(1-a^{2}\right)$.

\section{APPENDIX C: MODELING A ROUSE CHAIN IN A COILED TUBE}

The model describes the internal dynamics of the reptating chain as that of a one-dimensional Rouse chain, stretched so as to span the contour length of the tube. The potential energy takes the form

$$
\frac{\mathcal{V}}{k_{B} T}=\frac{1}{4 l^{2}} \sum_{j=1}^{N}\left(x_{j}-x_{j-1}\right)^{2}-\frac{h}{l}\left(x_{N}-x_{0}\right),
$$

where the $x_{j}, j=0, \ldots, N$ are the bead positions, $l$ measures the mean segment size, and $h / l$ is the stretching force acting on the end beads. The average extension of the chain is easily calculated as

$$
L=\left\langle x_{N}-x_{0}\right\rangle=2 \operatorname{lh} N
$$

The dynamics of the chain is given by a Langevin equation

$$
\frac{d}{d t} x_{j}=-\gamma_{0} \frac{\partial}{\partial x_{j}} \frac{\mathcal{V}}{k_{B} T}+\xi_{j}
$$

The fluctuating force $\xi_{j}$ is Gaussian distributed,

$$
\mathcal{P}\left[\xi_{j}(t)\right]=\mathcal{N}^{-1} \exp \left[-\frac{1}{4 \gamma_{0}} \int_{-\infty}^{+\infty} d t \xi_{j}^{2}(t)\right] .
$$

It is a standard exercise to calculate the dynamical structure functions. Indeed, for this system of coupled harmonic oscillators, the stretching force does not influence the dynamics but changes the static prefactor only. For the scattering from a pair $(j, k)$ of beads, one finds

$$
S^{(1 d)}(p, t ; j, k)=\left\langle\overline{e^{i p\left[x_{j}(t)-x_{k}(0)\right]}}\right\rangle=e^{2 i p l h(j-k)} e^{-p^{2} D_{j k}(t)},
$$

where

$$
\begin{aligned}
D_{j k}(t)= & \frac{\gamma_{0}|t|}{N+1}+l^{2}|j-k| \\
& +\frac{2 \gamma_{0}}{N+1} \sum_{\kappa=1}^{N} \cos \left(\pi \kappa \frac{j+\frac{1}{2}}{N+1}\right) \cos \left(\pi \kappa \frac{k+\frac{1}{2}}{N+1}\right) \\
& \times \frac{1-e^{-\omega_{\kappa}|t|}}{\omega_{\kappa}}, \\
\omega_{\kappa}= & 2 \frac{\gamma_{0}}{l^{2}} \sin ^{2} \frac{\pi \kappa}{2(N+1)} \approx \frac{\gamma_{0}}{2 l^{2}} \pi^{2} \frac{\kappa^{2}}{(N+1)^{2}}
\end{aligned}
$$

Using the approximate form of $\omega_{\kappa}$, we neglect some exponentially small microstructure effects.

In the analysis of Sec. V, we need this result for two segments deep inside a very long chain, for times small compared to the Rouse relaxation time of the total chain. Writing $j=N / 2+\widetilde{j}, k=N / 2+\widetilde{k}$ and taking the limit $N \rightarrow \infty$, with $\gamma_{0} t / l^{2} \gg 1$ fixed, one finds

$$
D_{j k}(t)=l^{2}|\tilde{j}-\tilde{k}|+l \sqrt{2 \gamma_{0} t} g\left(\frac{|\tilde{j}-\tilde{k}| l}{\sqrt{2 \gamma_{0} t}}\right),
$$

where

$$
g(z)=\frac{1}{\sqrt{\pi}} e^{-z^{2}-z \operatorname{erfc} z}
$$


Now assume that the chain is embedded in a coiled tube, consisting of $N / N_{e}$ segments of fixed length $l_{T}$. The contour length of the tube equals the length of the stretched chain,

$$
l_{T} \frac{N}{N_{e}}=L=2 \operatorname{lh} N .
$$

The end-to-end distance of the tube $R_{e}^{2}=l_{T}^{2}\left(N / N_{e}\right)$ must match the end-to-end distance of the physical chain. The potential energy of the latter is given by the threedimensional version of Eq. (C1) in the absence of the stretching force, which results in $R_{e}^{2}=6 l^{2} N$. Thus

$$
\frac{l_{T}^{2}}{l^{2}}=6 N_{e},
$$

and Eq. (C10) yields

$$
2 h=\sqrt{\frac{6}{N_{e}}} .
$$

To calculate the scattering from segments $j$ and $k$ of the stretched one-dimensional Rouse chain embedded in the tube, we write

$$
\begin{aligned}
S^{(R)}(q, t ; j, k) & =\left\langle\overline{e^{i \mathbf{q} \cdot\left[\mathbf{r}_{j}(t)-\mathbf{r}_{k}(0)\right]}}\right\rangle^{\text {Tube }}=\int_{-\infty}^{+\infty} d x\left\langle\overline{e^{i \mathbf{q} \cdot\left[\mathbf{r}_{j}(t)-\mathbf{r}_{k}(0)\right]} \delta\left(x_{j}(t)-x_{k}(0)-x\right)}\right\rangle^{\text {Tube }} \\
& =\left.\int_{-\infty}^{+\infty} d x\left\langle e^{i \mathbf{q} \cdot\left[\mathbf{r}_{j}(t)-\mathbf{r}_{k}(0)\right]}\right\rangle\right|_{x_{j}(t)-x_{k}(0)=x}\left\langle\overline{\delta\left(x_{j}(t)-x_{k}(0)-x\right)}\right\rangle .
\end{aligned}
$$

Here the first factor is to be calculated under the constraint that the points $\mathbf{r}_{j}(t)$ and $\mathbf{r}_{k}(0)$ have distance $x$ measured along the tube. It is thus given by the static correlation function of a chain of $x / l_{T}$ segments of fixed length $l_{T}$,

$$
\left.\left\langle e^{i \mathbf{q} \cdot\left[\mathbf{r}_{j}(t)-\mathbf{r}_{k}(0)\right]}\right\rangle\right|_{x_{j}(t)-x_{k}(0)=x}=e^{-\left(q^{2} / 6\right) l_{T}|x|} .
$$

The second factor in Eq. (C13) is the (one-dimensional) Fourier transform of $S^{(1 d)}$ [Eq. (C5)],

$$
\begin{aligned}
& \left\langle\overline{\delta\left(x_{j}(t)-x_{k}(0)-x\right)}\right\rangle \\
& \quad=\int_{-\infty}^{+\infty} \frac{d p}{2 \pi} e^{-i p x} S^{(1 d)}(p, t ; j, k) \\
& \quad=\left[4 \pi D_{j k}(t)\right]^{-1 / 2} \exp \left[-\frac{[x-2 h l(j-k)]^{2}}{4 D_{j k}(t)}\right] .
\end{aligned}
$$

Substituting Eqs. (C14) and (C15) into Eq. (C13), we can carry out the integral to find

$$
\begin{array}{rl}
S^{(R)}(q, t ; j, k)= & \frac{1}{2} e^{Q^{2}}\left\{e^{2 \Delta Q} \operatorname{erfc}(Q+\Delta)\right. \\
& \left.+e^{-2 \Delta Q} \operatorname{erfc}(Q-\Delta)\right\}, \\
Q=q^{2} & l \sqrt{\frac{N_{e}}{6} D_{j k}(t)}
\end{array}
$$

$$
\Delta=\frac{l(j-k)}{2 \sqrt{\frac{N_{e}}{6} D_{j k}(t)}} .
$$

If we take for $D_{j k}(t)$ the result (C8), the variables $Q$ and $\Delta$ reduce to $\widetilde{Q}$ and $\widetilde{\Delta}$ given in Eq. (5.15), and the result (C16) becomes identical to the expression (5.14).

A final remark on de Gennes' approximation [12] may be appropriate. The derivation starts from Eq. (C5) with $D_{j k}$ taken from Eq. (C8). Aiming directly at the coherent structure function, one integrates this expression over $j$ and $k$. If we ignore end effects, this yields

$$
\begin{aligned}
& \frac{1}{N} \int \operatorname{djdkS}^{(1 d)}(p, t ; j, k) \\
& \approx \int_{0}^{\infty} d s \exp \left[-p^{2} l^{2} s-p^{2} l \sqrt{2 \gamma_{0} t} g\left(\frac{s l}{\sqrt{2 \gamma_{0} t}}\right)\right] \\
& \quad \times\left(e^{2 i p l h s}+e^{-2 i p l h s}\right),
\end{aligned}
$$

where $s=|j-k|$. To evaluate this integral analytically, one expands $\exp \left[-p^{2} l \sqrt{2 \gamma_{0} t} g\left(s l / \sqrt{2 \gamma_{0} t}\right)\right]$ up to first order. The remaining steps closely follow our derivation given above and result in the form (2.12) of the "local" contribution to the coherent structure function. It should be noted that the expansion is valid only for $p^{2} l \sqrt{2 \gamma_{0} t} \ll 1$. The analysis supposes that $t$ is small compared to the Rouse relaxation time, and in this time regime, $\sqrt{2 \gamma_{0} t}$ is of the order of the mean square distance moved by a segment along the tube. Thus the condition $p^{2} l \sqrt{2 \gamma_{0} t} \approx p^{2}\left\langle\overline{\left(x_{j}(t)-x_{j}(0)\right)^{2}}\right\rangle \ll 1$ implies that the wave number $p$ cannot resolve the motion of a segment. 
[1] P. G. De Gennes, J. Chem. Phys. 55, 572 (1971).

[2] M. Doi and S. F. Edwards, The Theory of Polymer Dynamics (Clarendon Press, Oxford, 1986).

[3] K. S. Schweizer, M. Fuchs, G. Szamel, M. Guenza, and H. Tang, Macromol. Theory Simul. 6, 1037 (1997).

[4] W. Hess, Macromolecules 19, 1395 (1986).

[5] T. P. Lodge, N. A. Rotstein, and S. Prager, in Advances in Chemical Physics, Vol. 79, edited by I. Prigogine and Stuart A. Rice (Wiley, New York, 1990).

[6] See, e.g., A. N. Semenov, Sov. Phys. JETP 69, 107 (1989); B. H. Zimm and O. Lumpkin, Macromolecules 26, 226 (1993); A. N. Semenov and M. Rubinstein, Eur. Phys. J. B 1, 87 (1998).

[7] U. Ebert, A. Baumgärtner, and L. Schäfer, Phys. Rev. Lett. 78, 1592 (1997).

[8] U. Ebert, L. Schäfer, and A. Baumgärtner, J. Stat. Phys. 90, 1325 (1998).
[9] A. Baumgärtner, U. Ebert, and L. Schäfer, J. Stat. Phys. 90, 1375 (1998).

[10] T. Kreer, J. Baschnagel, M. Müller, and K. Binder, Macromolecules 34, 1105 (2001).

[11] M. Pütz, K. Kremer, and G. S. Grest, Europhys. Lett. 49, 735 (2000).

[12] P. G. de Gennes, J. Phys. (Paris) 42, 735 (1981).

[13] M. Doi and S. F. Edwards, J. Chem. Soc., Faraday Trans. 2 74, 1789 (1978).

[14] A. Baumgärtner, U. Ebert, and L. Schäfer, Macromolecules (to be published), http://xxx.lanl.gov/abs/cond-mat/0203218

[15] J. des Cloizeaux, J. Phys. I 3, 1523 (1993).

[16] J. des Cloizeaux, Macromolecules 23, 4678 (1990).

[17] K. E. Evans and S. F. Edwards, J. Chem. Soc., Faraday Trans. 2 77, 1891 (1981) 